Article

\title{
Genetic Parameter Estimates and Genotype $X$ Environment Interactions of Growth and Quality Traits for Betula alnoides Buch.-Ham. ex D. Don in Four Provenance-Family Trials in Southern China
}

\author{
Mingyu Yin, Junjie Guo *®D, Chunsheng Wang ${ }^{\circledR}$, Zhigang Zhao and Jie Zeng \\ Research Institute of Tropical Forestry, Chinese Academy of Forestry, Guangzhou 510520, China; \\ ymy920916@163.com (M.Y.); wang7760612@163.com (C.W.); zhaozhigang1979@126.com (Z.Z.); \\ zengj69@caf.ac.cn (J.Z.) \\ * Correspondence: guojunjie@caf.ac.cn; Tel.: +86-20-87030271
}

Received: 17 October 2019; Accepted: 12 November 2019; Published: 15 November 2019

\begin{abstract}
Betula alnoides is a valuable timber species with wide ecological adaptability in Southeast Asia and southern China. There are more than 150,000 ha of B. alnoides plantations in China until now. However, heavy differentiation in growth and quality traits with low productivity are usually seen in the practice. Elite germplasm are thus urgently needed for this species. Here growth and quality traits for 199 families of 25 provenances were assessed at four sites when 10-15 years old to estimate genetic parameters and reveal genotype by environment $(G \times E)$ interaction, and screen out superior provenances and families for plantation forestry of $B$. alnoides. The growth and quality performances of provenances varied with sites. Significant site, provenance, family and provenance-site effects were revealed for most growth and quality traits, while significant family-site effects were only observed in crown width (CW) and crown shape (CS), inferring that there existed significant $\mathrm{G} \times \mathrm{E}$ interaction. The provenance repeatability $\left(h_{p}^{2}\right)$ and family heritability $\left(h_{f}^{2}\right)$ of all tested traits ranged from 0.026 to 0.636 and 0.148 to 0.578 , respectively. Stem volume showed the highest genetic and phenotypic coefficients of variation (GCV, PCV) among all traits at each site. The diameter at breast height (DBH), tree height $(\mathrm{H})$, height to live crown base (HCB) and $\mathrm{CW}$ were strongly correlated with other traits, and were also under relatively higher genetic control and had stronger discriminating ability on genotype differences. Through biplot analyses of main genotypic effect and $\mathrm{G} \times \mathrm{E}$ interaction (GGE) for these traits, five provenances and 20 families were screened out with selection ratio of $20 \%$ and $10 \%$ at provenance and family level, respectively. The genetic and realized gains at provenance level ranged from $0.25 \%$ to $2.01 \%$ and $2.43 \%$ to $14.84 \%$, and those at family level ranged from $0.85 \%$ to $21.22 \%$ and $5.76 \%$ to $36.71 \%$, respectively. The findings lay a foundation for subsequent study on genetic improvement and breeding of this species, and application of the superior provenances and families will greatly increase the productivity of its plantations in practice.
\end{abstract}

Keywords: Betula alnoides; genetic variation; genotypes by environments interaction; genotypic correlation; heritability

\section{Introduction}

Betula alnoides Buch.-Ham. ex D. Don is a multiple-ploidy tree species in the genus Betula, family Betulaceae, and is indigenous to the warm subtropical and tropical regions in Southeast Asia and southern China [1]. B. alnoides is a fast-growing valuable tree species, its wood is widely used for floor and furniture-making as well as house decoration due to its beautiful texture, middle density, and excellent processing characteristics [2], and its barks are of medicinal application due to their 
anti-inflammatory and lipid-lowering properties [3,4]. In addition, B. alnoides is one of the pioneer tree species in evergreen broad-leaved secondary forests and its ecological values were also well recognized in water conservation, maintenance of biodiversity and soil fertility, and carbon sequestration [5]. This species has a wide adaptability to soil types, altitudes and climate conditions [6], and is becoming more and more popular in plantation forestry in southern China. Up to now, more than 150,000 ha of $B$. alnoides plantations have been established in Yunnan, Guangxi, Guangdong, and Fujian provinces [7]. However, heavy differentiation in growth and quality traits are usually observed in the practice due to the fact that plantations are mostly established with seedlings from unselected germplasms of this species. This leads to low productivity of the plantations. Consequently, the excellent germplasms have become in urgent need of plantation forestry of B. alnoides.

It is well-known that provenance or family tests are the foundation of selective breeding. Since 2000, many trial plantations have been established with provenances and families from natural distribution areas of Yunnan and Guangxi in order to screen out the excellent germplasm and broaden plantation scale of $B$. alnoides in southern China, which provide good support in genetic improvement and breeding of this species [5]. Based on early evaluations, many superior provenances and families were separately selected at each site in Yunnan, Guangxi, and Fujian [8-11]. These previous studies showed that the excellent provenances at one site might not maintain their good performance in other sites, which severely limits their application area. This difference may be attributed to the genotype by environment $(G \times E)$ interactions.

Genetic parameters like heritability, genetic correlation and genetic gain of traits are often used as a considerable basis for determining selection strategies and predicting selection effects, but $\mathrm{G} \times \mathrm{E}$ interaction usually affects the estimation of heritability and genetic gain in the breeding program of a tree species [12]. $G \times E$ interaction also reflects the adaptability of tree species to environmental factors such as air temperature, rainfall, soil, pests, etc. [13], and it has been reported for growth and quality traits as well as wood property of many important timber species. For example, significant $\mathrm{G} \times \mathrm{E}$ interactions were observed in tree height, diameter at breast height and volume for Betula pendula Roth, Betula kirghisorum Sav.-Ryczg, Betula pubescens Ehrh., Pinus densiflora Sieb. et Zucc. and Pinus taeda L. [14-16], in branchiness, straightness and wood density for Eucalyptus regnans F. Muell., Pinus radiate D. Don and Eucalyptus grandis W. Hill ex Maiden [17-19]. For B. alnoids, it is also essential to explain the pattern and range of $G \times E$ interaction by combined trials in multiple sites. This is useful to select germplasms which stably perform well across sites.

In the present study, four trial plantations of B. alnoides provenances and families are involved, which were established in Mengla and Changning, Yunnan, Pingxang, Guangxi and Hua'an, Fujian, respectively. Parts of these plantations were evaluated in the previous studies when one to four years old, and it was indicated that the variations among provenances and families were unstable and increased with age [9]. Now the ages of these plantations are 10 to 15 years old. The mean annual increments of tree height and diameter at breast height are the highest nearly in 15 years old which is about half a rotation and is also the appropriate age for combination test at multiple sites in terms of excellent germplasm selection [5]. The objectives of the present study were thus to (1) describe the variation of growth and quality traits in different sites; (2) estimate the genetic variation and heritability for these traits, and examine the genetic relationship among traits; (3) reveal the genotype by environment interactions for these traits; and (4) select the superior and stable provenances and families across sites. The findings will be helpful to improve the understanding of $G \times E$ interaction and promote the process of genetic improvement of this species.

\section{Materials and methods}

\subsection{Materials}

The Provenance-family trials of B. alnoides were conducted at four sites: Mengla, Yunnan; Pingxiang, Guangxi; Hua'an, Fujian; and Changning, Yunnan, where 400, 386, 280 and 250 half-sib 
families of all 25 provenances were involved, respectively. Only 199 half-sib families of 25 provenances existed in all four trials and were mainly analyzed in the present study. All the germplasms were collected from Yunnan and Guangxi, China. Table 1 shows the information of the four trials, and Table A1 shows the localities of 25 provenances. Randomized complete block design was used in each trial with single tree plots and 12 to 19 replicates. The seedlings were planted with $2 \mathrm{~m} \times 3 \mathrm{~m}$ spacing.

Table 1. Locations, climatic conditions, and tree age of four trials.

\begin{tabular}{ccccccc}
\hline Site & Latitude N & Longitude E & Altitude (m) & $\begin{array}{c}\text { Mean Annual Air } \\
\text { Temperature }\left({ }^{\circ} \mathbf{C}\right)\end{array}$ & $\begin{array}{c}\text { Rainfall } \\
\left(\mathbf{m m} \cdot \mathbf{Y e a r}^{-\mathbf{1})}\right.\end{array}$ & $\begin{array}{c}\text { Tree Age } \\
\left(\text { Year) }^{\prime}\right.\end{array}$ \\
\hline Mengla, Yunnan & $21^{\circ} 31^{\prime} 39^{\prime \prime}$ & $101^{\circ} 29^{\prime} 23^{\prime \prime}$ & 1161 & 21.70 & 1350 & 15 \\
Pingxiang, Guangxi & $22^{\circ} 01^{\prime} 59^{\prime \prime}$ & $106^{\circ} 51^{\prime} 01^{\prime \prime}$ & 550 & 20.20 & 1350 & 15 \\
Hua'an, Fujian & $24^{\circ} 56^{\prime} 19^{\prime \prime}$ & $117^{\circ} 30^{\prime} 59^{\prime \prime}$ & 275 & 20.80 & 1643 & 14 \\
Changning, Yunnan & $24^{\circ} 49^{\prime} 23^{\prime \prime}$ & $99^{\circ} 56^{\prime} 54^{\prime \prime}$ & 1250 & 18.50 & 1260 \\
\hline
\end{tabular}

\subsection{Measurement of Tree Growth and Quality Traits}

Stem diameter at breast height $(\mathrm{DBH})$ was measured using diameter tape with a precision of $0.1 \mathrm{~cm}$, tree height and height to live crown base (HCB) were measured using Vertex IV Altimeter (Haglöf Sweden AB, Västernorrland, Sverige) with a precision of $0.1 \mathrm{~m}$, and crown width $(\mathrm{CW})$ was measured using sliding staff with a precision of $0.1 \mathrm{~m}$. Stem volume (VOL) was calculated with the following equation [20]:

$$
V O L=\frac{0.45}{4} \times \pi D_{1.3}^{2} H
$$

where $D_{1.3}$ and $\mathrm{H}$ were stem diameter at breast height $(\mathrm{DBH})$ and tree height, respectively.

Quality traits including stem form (SF), crown shape (CS) and branchiness (BRA) were evaluated and standardized according to Wang et al. [21].

\subsection{Statistical Models and Genetic Analysis}

Variance analyses (ANOVA) and Duncan's multiple range tests were performed to estimate the variance of traits using univariate restricted maximum likelihood (REML) model in the Genstat 18th software. The following linear mixed models were used for individual site analysis (2) and multi-site analysis (3) at provenance level, respectively [22,23]:

$$
\begin{gathered}
y_{i j k}=\mu+B_{i}+P_{j}+P B_{i j}+e_{i j k} \\
y_{i j k l}=\mu+S_{i}+B(S)_{i j}+P_{k}+P S_{i k}+P B(S)_{i j k}+P Y S_{i k l}+P Y B(S)_{i j k l}+e_{i j k l m}
\end{gathered}
$$

where $y$ is the observations of traits, $\mu$ is the overall mean, $S$ and $B(S)$ are the fixed effects of site and block within site, $P, P S, P B(S), P Y S$ and $P Y B(S)$ are the random effects of provenance, and provenance-site, provenance-block within site, provenance-year-site, provenance-year-block within site interactions, respectively, and $e$ is residual error. The fixed effects of year and site-year and year-block within site interactions and the random effects of provenance-year interaction were excluded from the models (3) due to non-significant difference.

Provenance repeatability $\left(h_{p}^{2}\right)$ and variance components of phenotypic $\left(\sigma_{p h}^{2}\right)$ in individual and joint site analyses were estimated using Equations (4) and (6) as well as (5) and (7), respectively. Phenotypic coefficient of variation (PCV, \%) and genetic coefficient of variation (GCV, \%) were calculated with Equations (8) and (9), respectively [24]:

$$
h_{p}^{2}=\frac{\sigma_{p}^{2}}{\sigma_{p}^{2}+\frac{\sigma_{p b}^{2}}{n_{s} n_{b}}+\frac{\sigma_{e}^{2}}{n_{s} n_{b}}}
$$




$$
\begin{gathered}
h_{p}^{2}=\frac{\sigma_{p}^{2}}{\sigma_{p}^{2}+\frac{\sigma_{p s}^{2}}{n_{s}}+\frac{\sigma_{p b}^{2}}{n_{s} n_{b}}+\frac{\sigma_{p y s}^{2}}{n_{s} n_{y}}+\frac{\sigma_{p y b}^{2}}{n_{s} n_{b} n_{y}}+\frac{\sigma_{e}^{2}}{n_{s} n_{b} n_{y}}} \\
\sigma_{p h}^{2}=\sigma_{p}^{2}+\sigma_{p b}^{2}+\sigma_{e}^{2} \\
\sigma_{p h}^{2}=\sigma_{p}^{2}+\sigma_{p s}^{2}+\sigma_{p b}^{2}+\sigma_{p y s}^{2}+\sigma_{p y b}^{2}+\sigma_{e}^{2} \\
G C V=\frac{\sqrt{\sigma_{p}^{2}}}{\bar{X}} \times 100 \% \\
P C V=\frac{\sqrt{\sigma_{p h}^{2}}}{\bar{X}} \times 100 \%
\end{gathered}
$$

where $\sigma_{p}^{2}, \sigma_{p s}^{2}, \sigma_{p b}^{2}, \sigma_{p y s}^{2}, \sigma_{p y b}^{2}, \sigma_{e}^{2}, \bar{X}$ are the estimates of variance components for provenance, and provenance-site, provenance-block within site, provenance-year-site and provenance-year-block within site interactions, residual error and mean of traits; and $n_{s}, n_{y}, n_{b}$ are the number of sites, year and the harmonic mean of blocks within sites.

Family models for individual site analysis (10) and multiple sites joint analysis (11) were listed in the following [22,23]:

$$
\begin{gathered}
y_{i j k}=\mu+B_{i}+P_{j}+P B_{i j}+F(P)_{j k}+F(P) B_{i j k}+e_{i j k l} \\
y_{i j k}=\mu+S_{i}+B(S)_{i j}+P_{k}+P S_{i k}+P B(S)_{i j k}+P Y S_{i k l}+P Y B(S)_{i j k l}+ \\
F(P)_{k m}+F(P) Y_{k l m}+F(P) Y S_{i k l m}+F(P) Y B(S)_{i j k l m}+e_{i j k l m n}
\end{gathered}
$$

where $y$ is the observations of traits; $\mu$ is the overall mean; $S$ and $B(S)$ are the fixed effects of site and block within site; $P, F(P), P S, P B(S)$ and $F(P) S$ are the random effects of provenance, family, and provenance-site, provenance-block within site and family-site interactions, respectively; $F(P) B(S), P Y S$, $P Y B(S), F(P) Y S$ and $F(P) Y B(S)$ are the random effects of family-block within site, provenance-year-site, provenance-year-block within site, family-year-site and family-year-block within site interactions; and $e$ is residual error.

The fixed effects of year, and site-year and year-block within site interactions as well as the random effects of provenance-year, family-site and family-block within site interactions were excluded from the models due to non-significant difference.

Family heritability $\left(h_{f}^{2}\right)$ in individual site and multi-site analysis were estimated with the following Equations (12) and (13), respectively [24]:

$$
\begin{gathered}
h_{f}^{2}=\frac{\sigma_{f}^{2}}{\sigma_{f}^{2}+\frac{\sigma_{f b}^{2}}{n_{s} n_{b}}+\frac{\sigma_{e}^{2}}{n_{s} n_{b}}} \\
h_{f}^{2}=\frac{\sigma_{f}^{2}}{\sigma_{f}^{2}+\frac{\sigma_{f y}^{2}}{n_{y}}+\frac{\sigma_{f y s}^{2}}{n_{s} n_{y}}+\frac{\sigma_{f y b}^{2}}{n_{s} n_{b} n_{y}}+\frac{\sigma_{e}^{2}}{n_{s} n_{b} n_{y}}}
\end{gathered}
$$

where $\sigma_{f^{\prime}}^{2} \sigma_{f y^{\prime}}^{2} \sigma_{f y s^{\prime}}^{2} \sigma_{f y b^{\prime}}^{2} \sigma_{e}^{2}$ are variance components of family, and family-year, family-year-site and family-year-block within site interactions, and residual error of trait, respectively; and $n_{s}, n_{y}, n_{b}$ are the number of sites, year and the harmonic mean of blocks within sites. 
Genetic correlations $\left(r_{G}\right)$ among traits were calculated using the Equation (14) [25]:

$$
r_{G}=\frac{\operatorname{Cov}_{G}(x, y)}{\sqrt{\sigma_{G}^{2}(x) \times \sigma_{G}^{2}(y)}}
$$

where $\operatorname{Cov}_{G}(x, y)$ is the genotypic co-variances between trait $x$ and $y ; \sigma_{G}^{2}(x)$ and $\sigma_{G}^{2}(y)$ are the genotypic variances of trait $x$ and $y$.

The breeding values of provenances and families were estimated using the method of best linear unbiased prediction (BLUP), and were then used in biplot analysis for main genotypic effects and $\mathrm{G} \times \mathrm{E}$ interaction (GGE) by Genstat 18th software [26]. As described in detail, the breeding values of 25 provenances or 199 families in four sites could be displayed by a rank-two matrix. After the main environmental effects were removed through ANOVA, the main genotypic effect $(G)$ and interaction effect $(G \times E)$ were retained in the new matrix. The matrix was then decomposed into its component matrices using the method of singular-value decomposition. The first two principal components (PC1 and PC2) that explained about $85 \%$ of the total variation were used to build the biplot [27].

The superiority of provenances and families could be intuitively seen in the biplot graph according to their position in concentric circles, smaller concentric circle referred to better superiority. The superiority could also be ranked by superiority coefficients, smaller coefficients represented better superiority [12]. Based on the ranks of superiority, the desired provenances were then selected with the ratio being $20 \%$, and the excellent families were done with the ratio being $10 \%$ regardless of provenances.

Genetic gains $(\Delta G)$ and realized gains $\left(\Delta G_{r}\right)$ were calculated as follows [25]:

$$
\begin{gathered}
\Delta G=\frac{i h_{i}^{2} \sqrt{\sigma^{2}}}{\bar{X}} \times 100 \% \\
\Delta G_{r}=\frac{\bar{x}-\bar{X}}{\bar{X}} \times 100 \%
\end{gathered}
$$

where $i$ is selection intensity (the selection rate is $20 \%$ and $10 \%$ at levels of provenance and family, respectively); $h_{i}^{2}$ is heritability of trait; $\sqrt{\sigma^{2}}$ is standard deviation; $\bar{x}$ is mean of traits for selected provenances or families; and $\bar{X}$ is mean of traits for all provenances or families.

\section{Results}

\subsection{Variation of Growth and Quality Traits among Sites and Provenances}

Table 2 showed the means and ranges of growth and quality traits in each of the four trials. The trees at Mengla site showed the highest levels in the mean annual increment of tree height (MAH), crown width (CW) and branchiness (BRA), which indicated that Mengla was the most suitable for B. alnoids among the four sites. The mean annual increment of diameter at breast height (MABDH), mean annual increment of tree height (MAH), rate of height to live crown base to tree height (HCB.Height ${ }^{-1)}, \mathrm{CW}$ and mean annual increment of stem volume (MAVOL) were the lowest at Changning site. The trees at Hua' an site had the highest MADBH and MAVOL, but had the lowest stem form (SF) and BRA. 
Table 2. Growth and quality performance of Betula alnoids at four trial sites.

\begin{tabular}{|c|c|c|c|c|c|c|c|c|c|}
\hline Sites & Values & $\begin{array}{c}\text { MADBH } \\
\left(\mathrm{cm} \cdot \text { Year }^{-1}\right)\end{array}$ & $\begin{array}{c}\text { MAH } \\
\left(\mathrm{m} \cdot \text { Year-1) }^{-1}\right)\end{array}$ & HCB·Height ${ }^{-1}$ & $\mathrm{CW}(\mathrm{m})$ & $\begin{array}{c}\text { MAVOL } \\
\left(\mathrm{m}^{3} \cdot \text { Year }^{-1}\right)\end{array}$ & SF & CS & BRA \\
\hline \multirow[t]{2}{*}{ Mengla } & $\begin{array}{l}\text { Mean } \\
\text { (SE) }\end{array}$ & $1.34(0.01) b$ & $1.22(0.01) \mathrm{a}$ & $0.57(0.00) \mathrm{b}$ & $\begin{array}{c}4.71 \\
(0.04) \mathrm{a}\end{array}$ & $\begin{array}{c}0.0196 \\
(0.0003) \mathrm{a}\end{array}$ & $\begin{array}{c}0.63 \\
(0.02) \mathrm{c}\end{array}$ & $\begin{array}{c}0.65 \\
(0.01)\end{array}$ & $\begin{array}{c}0.77 \\
(0.01) \mathrm{a}\end{array}$ \\
\hline & Range & $0.3-2.5$ & $0.4-1.8$ & $0.1-0.9$ & $0.6-9.9$ & $0.000-0.069$ & $0.1-1.0$ & $0.1-1.0$ & $0.1-1.0$ \\
\hline \multirow[t]{2}{*}{ Pingxiang } & Mean & $1.04(0.01) \mathrm{c}$ & $0.94(0.01) \mathrm{c}$ & $0.61(0.01) \mathrm{a}$ & 4.49 & 0.0098 & 0.69 & 0.64 & 0.75 \\
\hline & Range & $0.3-2.5$ & $0.4-1.9$ & $0.2-0.9$ & $\begin{array}{l}\text { (0.06) D } \\
0.7-9.2\end{array}$ & $0.000-0.071$ & $\begin{array}{l}\text { (0.02) D } \\
0.1-1.0\end{array}$ & $0.1-1.0$ & $0.1-1.0$ \\
\hline Hua'an & Mean & $1.50(0.01) \mathrm{a}$ & $1.18(0.01) b$ & $0.56(0.00) c$ & 3.09 & 0.0204 & 0.52 & 0.64 & 0.65 \\
\hline \multirow{4}{*}{ Changning } & $\begin{array}{l}\text { Range } \\
\text { Ras }\end{array}$ & $0.3-2.5$ & $0.2-1.9$ & $0.2-1.4$ & $\begin{array}{l}\text { (0.05) C } \\
0.5-7.5\end{array}$ & $\begin{array}{c}(0.0003) \mathrm{a} \\
0.000-0.078\end{array}$ & $\begin{array}{l}\text { (0.01) d } \\
0.1-1.0\end{array}$ & $\begin{array}{c}(0.01) \\
0.1-1.0\end{array}$ & $\begin{array}{l}(0.01) \mathrm{C} \\
0.1-1.0\end{array}$ \\
\hline & Mean & ( 7801$) d(01)$ & $082(001) d$ & (0.01)d & 2.34 & 0.0020 & 0.77 & 0.64 & 0.73 \\
\hline & $(\mathrm{SE})$ & $0.78(0.01) \mathrm{d}$ & $0.83(0.01) \mathrm{d}$ & $0.51(0.01) \mathrm{d}$ & $(0.07) \mathrm{d}$ & $(0.0005) \mathrm{c}$ & $(0.03) \mathrm{a}$ & $(0.02)$ & $(0.01) \mathrm{b}$ \\
\hline & Range & $0.3-2.4$ & $0.4-1.9$ & $0.2-0.9$ & $0.6-6.7$ & $0.000-0.072$ & $0.1-1.0$ & $0.1-1.0$ & $0.1-1.0$ \\
\hline
\end{tabular}

Notes: MADBH, mean annual increment of diameter at breast height; MAH, mean annual increment of tree height; HCB.Height ${ }^{-1}$, rate of height to live crown base to tree height; CW, crown width; MAVOL, mean annual increment of stem volume; SF, stem form; CS, crown shape; BRA, branchiness. Means with standard error (SE) in parenthesis were of significant difference at 0.05 level according to Duncan's multiple range tests if followed by wholly different small letters in the same column.

Performance of growth and quality traits of 25 provenances were shown in Table 3, which were presented as means of all four sites for each trait. Significant differences were observed in all traits among provenances. MADBH, MAH, CW and MAVOL of provenance A were nearly the lowest in all 25 provenances, but its SF and BRA were the highest. The highest $\mathrm{MAH}$ and $\mathrm{CW}$ were observed in provenance $P$.

The growth and quality performance of 25 provenances differed among four sites (see Tables A2-A5). The lowest MAH, SF, CS, and BRA were observed in provenance $\mathrm{O}$ at the Mengla site. MADBH, MAH, MAVOL showed the best performance in provenance $\mathrm{V}$, while the poorest in provenance $\mathrm{L}$ at Pingxiang site. At Hua'an and Changning sites, the highest MADBH and MAVOL were seen in provenance $\mathrm{P}$, and the lowest MAH, MAVOL and SF in provenance $\mathrm{S}$.

\subsection{Genetic Variance, Heritability, and Genotypic Correlation}

It was shown from the estimation of variances and genetic parameters for all tested traits at each site (Table 4) that provenance effects were significant in most of the traits at four sites except SF and BRA at Pingxiang site as well as CS and BRA at Changning site. While significant family effects were mainly observed in growth traits at Mengla and Pingxiang sites. As a whole, the highest provenance repeatability $\left(h_{p}^{2}\right)$ of all tested traits were observed at Hua'an site (mean: 0.332), and the highest family heritability $\left(h_{f}^{2}\right)$ at Mengla site (mean: 0.164$)$. The genetic (GCV) and phenotypic (PCV) coefficient for variation of all traits at Hua' an site ranged from $3.15 \%$ to $15.93 \%$ and $20.30 \%$ to $70.44 \%$, with their means $(9.83 \%$ and $44.21 \%)$ being the highest in four sites. Volume and quality traits showed higher PCV than most of the other growth traits at each site.

The variances of sources, variance components, and genetic parameters were estimated at four sites together. Significant differences were observed only in CW and CS for family-site interaction, while for other sources of variances, significant differences were seen in most of the traits (Table 5 ). $h_{p}^{2}$ and $h_{f}^{2}$ of all traits ranged from 0.026 to 0.636 and 0.148 to 0.578 , respectively (Table 6). $h_{p}^{2}$ of HCB, SF and BRA showed higher levels than that of other traits. $h_{f}^{2}$ of growth, traits were higher than that of quality traits. GCV and PCV of variation ranged from $0.58 \%$ to $5.99 \%$ and $21.09 \%$ to $66.78 \%$, respectively. On the whole, GCV and PCV for quality traits were higher than those for most growth traits.

The genotypic correlation coefficients among all sorts of traits were shown in Table 7. There were moderate to strong positive genotypic correlations between DBH, height, and volume. Strong positive genotypic correlations were observed between $\mathrm{SF}, \mathrm{HCB}$, and $\mathrm{BRA}$, while strong negative correlations were seen between these traits and CW. 
Table 3. Growth and quality performance for 25 provenances of Betula alnoids.

\begin{tabular}{|c|c|c|c|c|c|c|c|c|}
\hline Provenances & MADBH $\left(\mathrm{cm} \cdot\right.$ Year $\left.^{-1}\right)$ & MAH $\left(m \cdot\right.$ Year $\left.^{-1}\right)$ & HCW·Height ${ }^{-1}$ & $\mathrm{CW}(\mathrm{m})$ & MAVOL $\left(m^{3} \cdot\right.$ Year $\left.^{-1}\right)$ & SF & CS & BRA \\
\hline A & $1.04(0.05) \mathrm{j}$ & $1.02(0.05) \mathrm{f}$ & $0.60(0.02) \mathrm{ab}$ & $3.16(0.25) \mathrm{c}$ & $0.0096(0.0013) \mathrm{f}$ & $0.77(0.04) \mathrm{a}$ & $0.66(0.05) b c$ & $0.83(0.04) \mathrm{a}$ \\
\hline B & $1.29(0.05)$ abcde & $1.08(0.04)$ bcdef & 0.57 (0.02) bcdefgh & $3.80(0.23) \mathrm{ab}$ & $0.0158(0.0017)$ abcde & $0.67(0.04)$ bcde & $0.66(0.04) b c$ & $0.73(0.04)$ bcdef \\
\hline $\mathrm{C}$ & $1.19(0.03)$ bcdefghi & $1.12(0.02)$ abcde & $0.61(0.01) \mathrm{a}$ & $3.68(0.14) \mathrm{ab}$ & 0.0148 (0.0011) abcde & $0.63(0.03)$ cdefg & $0.63(0.03) b c$ & $0.77(0.02)$ abcde \\
\hline $\mathrm{D}$ & $1.12(0.04) \mathrm{ij}$ & $1.04(0.03)$ ef & $0.59(0.01)$ abcde & $3.72(0.18) \mathrm{ab}$ & $0.0122(0.0011) \mathrm{def}$ & $0.68(0.03)$ bcde & $0.67(0.03) b c$ & $0.76(0.03)$ bcdef \\
\hline $\mathrm{F}$ & $1.15(0.06)$ fghij & $1.05(0.04)$ def & $0.58(0.02)$ bcdef & $3.28(0.22) b c$ & $0.0133(0.0018)$ cdef & $0.75(0.04) \mathrm{abc}$ & $0.63(0.04) b c$ & $0.82(0.03) \mathrm{ab}$ \\
\hline G & $1.18(0.07)$ cdefghi & $1.13(0.05)$ abcde & $0.60(0.02) \mathrm{abcd}$ & $3.49(0.25) \mathrm{abc}$ & $0.0183(0.0023) \mathrm{a}$ & $0.70(0.05) \mathrm{abcd}$ & $0.69(0.05) b c$ & $0.80(0.04) \mathrm{ab}$ \\
\hline $\mathrm{H}$ & $1.25(0.04)$ abcdefgh & $1.11(0.03)$ abcde & $0.58(0.01)$ bcdefg & $3.97(0.18)$ a & $0.0156(0.0011)$ abcde & $0.69(0.03)$ bcde & $0.66(0.03) \mathrm{bc}$ & 0.77 (0.03) abcde \\
\hline I & $1.20(0.05)$ bcdefghi & $1.14(0.03) \mathrm{abc}$ & $0.58(0.01)$ abcdef & $3.94(0.18) \mathrm{a}$ & $0.0166(0.0014)$ abcd & $0.67(0.03)$ bcdef & $0.64(0.04) \mathrm{bc}$ & $0.74(0.03)$ bcdef \\
\hline $\mathrm{J}$ & $1.21(0.03)$ bcdefghi & $1.11(0.02)$ abcde & $0.60(0.01) \mathrm{ab}$ & $3.84(0.15) \mathrm{ab}$ & $0.0150(0.001)$ abcde & $0.64(0.03)$ bcdefg & $0.58(0.03) \mathrm{c}$ & $0.79(0.03) \mathrm{abc}$ \\
\hline $\mathrm{L}$ & $1.15(0.04)$ fghij & $1.07(0.03)$ cdef & 0.57 (0.01) bcdefgh & $3.78(0.15) \mathrm{ab}$ & 0.0141 (0.0012) abcde & $0.75(0.02) \mathrm{ab}$ & $0.62(0.03) \mathrm{bc}$ & $0.79(0.03) \mathrm{abc}$ \\
\hline $\mathrm{M}$ & 1.17 (0.04) efghi & $1.08(0.03)$ bcdef & $0.60(0.01) \mathrm{abc}$ & $3.35(0.18) a b c$ & $0.0137(0.0012)$ bcdef & $0.69(0.03)$ bcde & $0.59(0.04) \mathrm{bc}$ & $0.78(0.03)$ abcde \\
\hline $\mathrm{N}$ & $1.12(0.04)$ hij & $1.03(0.03)$ ef & $0.55(0.01)$ cdefgh & $3.72(0.15) \mathrm{ab}$ & $0.0127(0.0013)$ cdef & $0.61(0.03)$ defg & $0.62(0.03) \mathrm{bc}$ & $0.71(0.03)$ bcdef \\
\hline $\mathrm{O}$ & $1.18(0.03)$ defghi & $1.06(0.03) \mathrm{def}$ & $0.57(0.01)$ bcdefgh & $3.61(0.14) \mathrm{ab}$ & $0.0128(0.0008)$ cdef & $0.60(0.030)$ defgh & $0.61(0.030) \mathrm{bc}$ & $0.67(0.03)$ cdef \\
\hline $\mathrm{P}$ & $1.33(0.04) \mathrm{abc}$ & $1.16(0.02) \mathrm{a}$ & $0.53(0.01) \mathrm{h}$ & $3.91(0.14)$ a & $0.0186(0.0013) \mathrm{a}$ & $0.66(0.026)$ bcdefg & $0.71(0.03) \mathrm{b}$ & $0.69(0.03) \mathrm{cdef}$ \\
\hline $\mathrm{Q}$ & $1.25(0.05)$ abcdefgh & $1.07(0.03)$ bcdef & $0.53(0.02) \mathrm{h}$ & $3.90(0.20) \mathrm{ab}$ & $0.0140(0.0013)$ bcdef & $0.59(0.04)$ efgh & $0.86(0.03) \mathrm{a}$ & $0.66(0.05) \mathrm{ef}$ \\
\hline $\mathrm{R}$ & $1.33(0.03) \mathrm{ab}$ & $1.11(0.02)$ abcde & $0.53(0.01) \mathrm{gh}$ & $3.85(0.12) \mathrm{ab}$ & $0.0175(0.0009) \mathrm{ab}$ & $0.58(0.02)$ efgh & $0.67(0.02) \mathrm{bc}$ & $0.66(0.02) \mathrm{f}$ \\
\hline $\mathrm{s}$ & $1.15(0.04)$ ghij & $1.01(0.04) \mathrm{f}$ & $0.57(0.02)$ bcdefg & $3.21(0.21) b c$ & $0.0116(0.0011)$ ef & $0.49(0.05) \mathrm{h}$ & $0.64(0.04) \mathrm{bc}$ & $0.70(0.05)$ bcdef \\
\hline $\mathrm{T}$ & $1.26(0.03)$ abcdefg & $1.08(0.02)$ bcdef & $0.55(0.01)$ efgh & $3.61(0.14) \mathrm{abc}$ & $0.0145(0.0009)$ abcde & $0.55(0.03) \mathrm{fgh}$ & $0.64(0.03) \mathrm{bc}$ & $0.67(0.03)$ cdef \\
\hline $\mathrm{U}$ & $1.23(0.03)$ bcdefgh & $1.07(0.02)$ bcdef & $0.55(0.01)$ efgh & $3.76(0.11) \mathrm{ab}$ & $0.0150(0.0008)$ abcde & $0.59(0.02)$ defgh & $0.65(0.02) \mathrm{bc}$ & $0.66(0.02)$ ef \\
\hline $\mathrm{V}$ & $1.28(0.04)$ abcdef & $1.07(0.03)$ bcdef & $0.54(0.01) \mathrm{fgh}$ & $3.74(0.18) \mathrm{ab}$ & 0.0149 (0.0011) abcde & $0.63(0.03)$ bcdefg & $0.71(0.03) \mathrm{bc}$ & $0.73(0.03)$ bcdef \\
\hline Y & $1.31(0.02)$ abcd & $1.13(0.02) \mathrm{abcd}$ & $0.56(0.01)$ bcdefgh & $3.99(0.10) \mathrm{a}$ & $0.0166(0.0007) a b c$ & $0.60(0.02)$ defg & $0.71(0.02) \mathrm{b}$ & $0.74(0.02)$ bcdef \\
\hline Means & $1.25(0.01)$ & $1.09(0.01)$ & $0.56(0.00)$ & $3.78(0.03)$ & $0.0154(0.0002)$ & $0.62(0.01)$ & $0.66(0.01)$ & $0.72(0.01)$ \\
\hline
\end{tabular}

Notes: A, Mengla; B, Yuanyang; C, Mojiang; D, Jinghong; E, Xichou; F, Zhenyuan; G, Tengchong; H, Jinggu; I, Ruili; J, Fengqing; K, Pingbian; L, Jiangcheng; M, Shuangiiang; N, Lancang; O, Lingyun; P, Longzhou; Q, Donglan; R, Tianlin; S, Debao; T, Tiane; U, Pingguo; V, Baise; W, Tianyang; X, Jingxi; Y, Napo; MADBH, mean annual increment of diameter at breast height; $\mathrm{MAH}$, mean annual increment of tree height; HCB.Height ${ }^{-1}$, rate of height to live crown base to tree height; CW, crown width; MAVOL, mean annual increment of stem volume; SF, stem form; CS, crown shape; BRA, branchiness. Means with standard error in parenthesis were of significant difference at 0.05 level according to Duncan's multiple range tests if followed by wholly different small letters in the same column. 
Table 4. Estimates of variances $\left(\sigma_{p}^{2}, \sigma_{p b^{\prime}}^{2} \sigma_{f}^{2}, \sigma_{f b^{\prime}}^{2}, \sigma_{e}^{2}, \sigma_{p h}^{2}\right)$ for provenance, provenance-block within site, family, family-block within site, residual error and phenotypic variance, provenance repeatability $\left(h_{p}^{2}\right)$, family heritability $\left(h_{f}^{2}\right)$, co-efficient of variation at genetic and phenotypic level (genetic coefficient of variation (GCV), phenotypic coefficient of variation (PCV)) for tested traits at each site.

\begin{tabular}{|c|c|c|c|c|c|c|c|c|c|c|c|c|c|c|}
\hline Sites & Traits & $F$ Value of $\sigma_{p}^{2}$ & $F$ Value of $\sigma_{f}^{2}$ & $\sigma_{p}^{2}$ & $\sigma_{p b}^{2}$ & $\sigma_{e}^{2}$ & $h_{p}^{2}$ & $\sigma_{f}^{2}$ & $\sigma_{f b}^{2}$ & $\sigma_{e}^{2}$ & $h_{f}^{2}$ & $\sigma_{p h}^{2}$ & GCV\% & PCV\% \\
\hline \multirow[t]{8}{*}{ Mengla } & DBH & $2.50 * * *$ & $1.30 *$ & 0.647 & 0.287 & 23.530 & $0.230(0.096)$ & 1.297 & $\mathrm{NE}$ & 22.250 & $0.226(0.079)$ & 24.464 & 4.00 & 24.58 \\
\hline & Height & $2.74^{* * *}$ & $1.34^{* *}$ & 0.392 & 0.283 & 12.560 & $0.252(0.100)$ & 0.592 & $\mathrm{NE}$ & 11.960 & $0.198(0.084)$ & 13.236 & 3.40 & 19.75 \\
\hline & HCB & $4.03 * * *$ & $1.22 *$ & 0.336 & 0.893 & 6.955 & $0.320(0.114)$ & 0.355 & 4.165 & 2.475 & $0.211(0.084)$ & 8.184 & 5.54 & 27.37 \\
\hline & CW & $2.27^{* * *}$ & $1.29 *$ & 0.042 & 0.102 & 2.191 & $0.169(0.098)$ & 0.127 & 0.833 & 1.232 & $0.2350 .079)$ & 2.335 & 4.35 & 32.29 \\
\hline & Volume & $2.60 * * *$ & $1.36^{* *}$ & 0.001 & 0.001 & 0.030 & $0.236(0.098)$ & 0.002 & $\mathrm{NE}$ & 0.028 & $0.246(0.079)$ & 0.031 & 9.83 & 59.43 \\
\hline & SF & $2.54^{* * *}$ & $1.20 \mathrm{~ns}$ & 0.002 & 0.006 & 0.089 & $0.199(0.108)$ & 0.003 & $\mathrm{NE}$ & 0.086 & $0.160(0.085)$ & 0.097 & 7.32 & 49.34 \\
\hline & CS & $2.62 * * *$ & $1.10 \mathrm{~ns}$ & 0.003 & 0.008 & 0.095 & $0.211(0.109)$ & 0.001 & 0.004 & 0.091 & $0.029(0.095)$ & 0.105 & 7.62 & 49.48 \\
\hline & BRA & $2.18^{* * *}$ & $0.86 \mathrm{~ns}$ & 0.002 & 0.005 & 0.091 & $0.184(0.105)$ & 0.000 & $\mathrm{NE}$ & 0.091 & $0.006(0.064)$ & 0.098 & 5.73 & 40.42 \\
\hline \multirow[t]{8}{*}{ Pingxiang } & DBH & $8.69 * * *$ & $1.33 *$ & 3.084 & 2.773 & 13.080 & 0.609 (0.094) & 0.435 & 13.000 & 0.285 & $0.090(0.109)$ & 18.937 & 11.17 & 27.67 \\
\hline & Height & $5.50 * * *$ & $1.25 \mathrm{~ns}$ & 0.620 & 3.018 & 7.069 & $0.330(0.147)$ & 0.124 & 7.318 & 0.045 & $0.048(0.121)$ & 10.707 & 5.59 & 23.23 \\
\hline & $\mathrm{HCB}$ & $3.11 * * *$ & $1.50^{* * * *}$ & 0.044 & 0.997 & 2.531 & $0.091(0.154)$ & 0.012 & 2.489 & 0.095 & $0.014(0.121)$ & 3.572 & 2.52 & 22.62 \\
\hline & CW & $4.46^{* * *}$ & $1.60^{* * *}$ & 0.230 & 0.479 & 2.616 & $0.373(0.128)$ & 0.093 & 2.409 & 0.183 & $0.098(0.116)$ & 3.325 & 10.66 & 40.52 \\
\hline & Volume & $5.45^{* * *}$ & $1.12 \mathrm{~ns}$ & 0.001 & 0.002 & 0.009 & $0.480(0.117)$ & 0.000 & 0.009 & 0.000 & $0.093(0.109)$ & 0.012 & 23.39 & 72.69 \\
\hline & SF & $0.78 \mathrm{~ns}$ & $0.98 \mathrm{~ns}$ & 0.000 & 0.007 & 0.082 & $0.001(0.092)$ & 0.002 & 0.080 & 0.006 & $0.055(0.101)$ & 0.088 & 0.46 & 42.92 \\
\hline & CS & $4.67 * * *$ & $1.59 * * *$ & 0.008 & 0.013 & 0.084 & $0.384(0.127)$ & 0.002 & 0.081 & 0.005 & $0.072(0.112)$ & 0.104 & 13.46 & 50.12 \\
\hline & BRA & $1.44 \mathrm{~ns}$ & $1.11 \mathrm{~ns}$ & 0.001 & 0.009 & 0.079 & $0.057(0.114)$ & 0.000 & 0.080 & 0.004 & $0.003(0.106)$ & 0.089 & 3.45 & 39.82 \\
\hline \multirow[t]{8}{*}{ Hua'an } & DBH & $3.60^{* * *}$ & $0.94 \mathrm{~ns}$ & 1.120 & 1.080 & 21.080 & $0.336(0.107)$ & 0.020 & 2.239 & 18.950 & $0.004(0.071)$ & 23.280 & 5.01 & 22.85 \\
\hline & Height & $2.50 * * *$ & $1.14 \mathrm{~ns}$ & 0.270 & 0.815 & 10.140 & $0.197(0.112)$ & 0.274 & 0.475 & 9.445 & $0.099(0.075)$ & 11.224 & 3.15 & 20.30 \\
\hline & $\mathrm{HCB}$ & $3.19^{* * *}$ & $1.18 \mathrm{~ns}$ & 0.296 & 0.326 & 5.581 & $0.334(0.112)$ & 0.005 & 0.643 & 4.959 & $0.003(0.078)$ & 6.203 & 6.07 & 27.79 \\
\hline & CW & $3.52 * * *$ & $0.94 \mathrm{~ns}$ & 0.106 & 0.081 & 1.943 & $0.343(0.107)$ & 0.002 & 0.187 & 1.768 & $0.003(0.076)$ & 2.129 & 10.28 & 46.16 \\
\hline & Volume & $4.01^{* * *}$ & $1.10 \mathrm{~ns}$ & 0.002 & 0.001 & 0.026 & $0.363(0.105)$ & 0.000 & 0.002 & 0.024 & $0.028(0.076)$ & 0.029 & 13.76 & 59.23 \\
\hline & SF & $2.54^{* * *}$ & $0.97 \mathrm{~ns}$ & 0.005 & 0.000 & 0.121 & $0.275(0.104)$ & 0.000 & 0.011 & 0.111 & $0.001(0.070)$ & 0.126 & 13.48 & 70.44 \\
\hline & CS & $4.83^{* * *}$ & $1.41 * * *$ & 0.011 & $\mathrm{NE}$ & 0.109 & $0.509(0.097)$ & 0.004 & 0.025 & 0.082 & $0.119(0.078)$ & 0.121 & 15.93 & 51.96 \\
\hline & BRA & $3.25 * * *$ & $1.00 \mathrm{~ns}$ & 0.005 & 0.008 & 0.108 & $0.294(0.109)$ & 0.000 & 0.015 & 0.094 & $0.014(0.069)$ & 0.121 & 10.97 & 54.90 \\
\hline \multirow{8}{*}{ Changning } & DBH & $2.86^{* * *}$ & $1.02 \mathrm{~ns}$ & 0.215 & 0.130 & 2.835 & $0.567(0.126)$ & 0.042 & 0.710 & 2.097 & $0.057(0.143)$ & 3.180 & 6.01 & 23.08 \\
\hline & Height & $1.83 *$ & $0.97 \mathrm{~ns}$ & 0.096 & 0.064 & 2.695 & $0.386(0.168)$ & 0.132 & $\mathrm{NE}$ & 2.577 & $0.170(0.123)$ & 2.855 & 3.74 & 20.36 \\
\hline & HCB & $1.58^{*}$ & $1.09 \mathrm{~ns}$ & 0.010 & 0.137 & 1.270 & $0.108(0.256)$ & 0.065 & 0.201 & 1.003 & $0.178(0.125)$ & 1.416 & 2.31 & 28.18 \\
\hline & CW & $1.99 * *$ & $1.38 *$ & 0.027 & $\mathrm{NE}$ & 0.653 & $0.425(0.153)$ & 0.059 & $\mathrm{NE}$ & 0.601 & $0.282(0.104)$ & 0.680 & 6.94 & 34.98 \\
\hline & Volume & $2.21 *$ & $0.97 \mathrm{~ns}$ & 0.000 & 0.000 & 0.000 & $0.460(0.154)$ & 0.000 & 0.000 & 0.000 & $0.081(0.139)$ & 0.000 & 13.16 & 61.93 \\
\hline & SF & $2.21 * * *$ & $0.97 \mathrm{~ns}$ & 0.000 & 0.003 & 0.068 & $0.060(0.122)$ & 0.000 & 0.001 & 0.065 & $0.002(0.112)$ & 0.071 & 2.06 & 34.87 \\
\hline & CS & $0.93 \mathrm{~ns}$ & $0.85 \mathrm{~ns}$ & 0.000 & 0.002 & 0.116 & $0.027(0.229)$ & 0.000 & 0.022 & 0.094 & $0.001(0.105)$ & 0.118 & 2.09 & 52.92 \\
\hline & BRA & $0.69 \mathrm{~ns}$ & $1.15 \mathrm{~ns}$ & 0.000 & $\mathrm{NE}$ & 0.090 & 0.019 (0.151) & 0.003 & $\mathrm{NE}$ & 0.087 & $0.116(0.121)$ & 0.090 & 0.43 & 41.11 \\
\hline
\end{tabular}

Notes: DBH, diameter at breast height; $\mathrm{HCB}$, height to live crown base; $\mathrm{CW}$, crown width; $\mathrm{SF}$, stem form; CS, crown shape; BRA, branchiness. Features in parentheses were standard errors. ${ }^{* * *} p<0.001 ;{ }^{* *} 0.001<p<0.01 ; * 0.01<p<0.05 ;$ ns indicates not significant, level of significance of effects. NE, not estimated and assumed to be zero. 
Table 5. F values and significance levels for variance from different sources of all traits in combined variance analysis of four sites.

\begin{tabular}{|c|c|c|c|c|c|c|c|}
\hline Traits & Site (3) & $\begin{array}{c}\text { Block } \\
\text { within } \\
\text { Site }(48)\end{array}$ & $\begin{array}{l}\text { Provenance } \\
\text { (24) }\end{array}$ & $\begin{array}{c}\text { Provenance-Site } \\
\text { (72) }\end{array}$ & $\begin{array}{l}\text { Provenance-Block } \\
\text { within Site (801) }\end{array}$ & $\begin{array}{l}\text { Family } \\
\text { (199) }\end{array}$ & $\begin{array}{c}\text { Family-Site } \\
(388)\end{array}$ \\
\hline $\mathrm{DBH}$ & $1517.82^{* * *}$ & $6.25^{* * *}$ & $3.71 * * *$ & $3.27^{* * *}$ & $1.10 *$ & $1.35^{* *}$ & $0.93 \mathrm{~ns}$ \\
\hline Height & $1560.85^{* * *}$ & $6.98^{* * *}$ & $2.73^{* * *}$ & $2.66^{* * *}$ & $1.30^{* * *}$ & $1.46^{* * *}$ & $1.01 \mathrm{~ns}$ \\
\hline НCB & $1073.10^{* * *}$ & $13.06^{* * *}$ & $5.16^{* * *}$ & $1.90^{* * *}$ & $1.40^{* * *}$ & $1.39^{* * *}$ & $0.96 \mathrm{~ns}$ \\
\hline CW & $549.52^{* * *}$ & $5.72^{* * *}$ & $3.01 * * *$ & $3.03^{* * *}$ & $1.30^{* * *}$ & $1.41^{* * *}$ & $1.24^{* *}$ \\
\hline Volume & $625.06^{* * *}$ & $4.93^{* * *}$ & $3.17 * * *$ & $2.60^{* * *}$ & $1.02 \mathrm{~ns}$ & $1.39^{* * *}$ & $0.88 \mathrm{~ns}$ \\
\hline SF & $111.01^{* * *}$ & $6.12^{* * *}$ & $3.23 * * *$ & $1.20 \mathrm{~ns}$ & $1.10 \mathrm{~ns}$ & $1.16 \mathrm{~ns}$ & $0.92 \mathrm{~ns}$ \\
\hline $\mathrm{CS}$ & $1.01 \mathrm{~ns}$ & $6.22 * * *$ & $2.88^{* * *}$ & $3.06^{* * *}$ & $1.23^{* * *}$ & $1.34^{* *}$ & $1.24^{* *}$ \\
\hline BRA & $45.03 * * *$ & $4.15^{* * *}$ & $3.45^{* * *}$ & $1.37^{*}$ & $1.20^{* * *}$ & $1.05 \mathrm{~ns}$ & $0.89 \mathrm{~ns}$ \\
\hline
\end{tabular}

Notes: DBH, diameter at breast height; $\mathrm{HCB}$, height to live crown base; $\mathrm{CW}$, crown width; $\mathrm{SF}$, stem form; $\mathrm{CS}$, crown shape; BRA, branchiness. The number in parentheses is the degree of freedom. ${ }^{* * *} p<0.001$; ${ }^{* *} 0.001<p<0.01$; $* 0.01<p<0.05$; ns indicates not significant, level of significance of effects.

Table 6. Estimates of variances $\left(\sigma_{p}^{2}, \sigma_{p s^{\prime}}^{2}, \sigma_{p b^{\prime}}^{2} \sigma_{p y s}^{2}, \sigma_{p y b^{\prime}}^{2} \sigma_{f}^{2}, \sigma_{f y^{\prime}}^{2}, \sigma_{f y s^{\prime}}^{2}, \sigma_{f y b^{\prime}}^{2}, \sigma_{e}^{2}, \sigma_{p h}^{2}\right)$ for provenance, provenance-site, provenance-block within site, provenance-year-site, provenance-year-block within site, family, family-year, family-year-site, family-year-block within site, residual error and phenotypic variance, provenance repeatability $\left(h_{p}^{2}\right)$, family heritability $\left(h_{f}^{2}\right)$, coefficient of variation at genetic and phenotypic level (GCV, PCV) for various traits in combined analysis of four sites.

\begin{tabular}{ccccccccc}
\hline Random Effect & DBH & Height & HCB & CW & Volume & SF & CS & BRA \\
\hline$\sigma_{p}^{2}$ & 0.080 & 0.008 & 0.091 & 0.007 & 0.000 & 0.001 & 0.000 & 0.001 \\
$\sigma_{p s}^{2}$ & $\mathrm{NE}$ & $\mathrm{NE}$ & 0.099 & $\mathrm{NE}$ & 0.001 & 0.001 & 0.005 & $\mathrm{NE}$ \\
$\sigma_{p b}^{2}$ & $\mathrm{NE}$ & 0.885 & $\mathrm{NE}$ & 0.136 & 0.001 & $\mathrm{NE}$ & 0.005 & $\mathrm{NE}$ \\
$\sigma_{p y s}^{2}$ & 1.060 & 0.349 & $\mathrm{NE}$ & 0.085 & $\mathrm{NE}$ & $\mathrm{NE}$ & $\mathrm{NE}$ & 0.001 \\
$\sigma_{p y b}^{2}$ & 0.900 & $\mathrm{NE}$ & 0.574 & $\mathrm{NE}$ & $\mathrm{NE}$ & 0.004 & $\mathrm{NE}$ & 0.006 \\
$\sigma_{e}^{2}$ & 17.590 & 9.225 & 4.816 & 1.948 & 0.020 & 0.095 & 0.102 & 0.094 \\
$h_{p}^{2}$ & 0.276 & 0.066 & 0.602 & 0.230 & 0.026 & 0.636 & 0.123 & 0.634 \\
$\sigma_{f}^{2}$ & $(0.581)$ & $(0.043)$ & $(0.160)$ & $(0.396)$ & $(0.037)$ & $(0.147)$ & $(0.114)$ & $(0.115)$ \\
$\sigma_{f y}^{2}$ & 0.193 & 0.147 & 0.112 & 0.029 & 0.001 & 0.001 & 0.000 & 0.000 \\
$\sigma_{f y s}^{2}$ & 0.146 & 0.090 & $\mathrm{NE}$ & $\mathrm{NE}$ & $\mathrm{NE}$ & $\mathrm{NE}$ & $\mathrm{NE}$ & $\mathrm{NE}$ \\
$\sigma_{f y b}^{2}$ & 0.005 & $\mathrm{NE}$ & 0.041 & 0.037 & $\mathrm{NE}$ & $\mathrm{NE}$ & 0.002 & $\mathrm{NE}$ \\
$\sigma_{e}^{2}$ & 1.393 & 4.730 & 1.133 & 0.558 & 0.002 & 0.018 & 0.035 & 0.022 \\
$h_{f}^{2}$ & 8.015 & 4.730 & 3.849 & 1.439 & 0.019 & 0.080 & 0.065 & 0.074 \\
$\sigma_{p h}^{2}$ & 0.428 & 0.276 & 0.495 & 0.379 & 0.578 & 0.173 & 0.149 & 0.148 \\
$\mathrm{GCV} \%$ & $(0.099)$ & $(0.152)$ & $(0.124)$ & $(0.151)$ & $(0.083)$ & $(0.153)$ & $(0.136)$ & $(0.161)$ \\
$\mathrm{PCV} \%$ & 19.630 & 10.467 & 5.580 & 2.176 & 0.022 & 0.100 & 0.112 & 0.102 \\
\end{tabular}

Notes: DBH, diameter at breast height; $\mathrm{HCB}$, height to live crown base; $\mathrm{CW}$, crown width; $\mathrm{SF}$, stem form; $\mathrm{CS}$, crown shape; BRA, branchiness. The variance analysis at provenance level was estimated in all four sites, while that at family level was estimated in Mengla, Pingxiang and Huanan sites, and the values shown in parentheses were standard errors. NE, not estimated and assumed to be zero.

Table 7. Genotypic correlation coefficients among tested traits.

\begin{tabular}{cccccccc}
\hline Traits & Height & HCB & CW & Volume & SF & CS & BRA \\
\hline DBH & $0.440^{*}$ & -0.386 & $0.765^{* *}$ & $0.732^{* *}$ & $-0.493^{*}$ & $0.657^{*}$ & $-0.520^{*}$ \\
Height & & $0.580^{*}$ & -0.117 & $0.521^{*}$ & 0.386 & 0.028 & $0.430^{*}$ \\
HCB & & & $-0.795^{* *}$ & -0.088 & $0.705^{* *}$ & $-0.595^{*}$ & $0.922^{* *}$ \\
CW & & & & $0.482^{*}$ & $-0.701^{* *}$ & $0.807^{*}$ & $-0.776^{* *}$ \\
Volume & & & & & 0.006 & $0.547^{*}$ & -0.041 \\
SF & & & & & & $-0.476^{*}$ & $0.855^{* *}$ \\
CS & & & & & & & $-0.531^{*}$
\end{tabular}

Notes: $\overline{\mathrm{DBH}}$, diameter at breast height; $\mathrm{HCB}$, height to live crown base; $\mathrm{CW}$, crown width; SF, stem form; $\mathrm{CS}$, crown shape; BRA, branchiness. ${ }^{* *} p<0.01 ;{ }^{*} 0.01<p<0.05$, level of significance of correlation coefficients.

\subsection{Genotype by Environment $(G \times E)$ Interaction}

Provenance-site interactions were significant for all traits except SF, while family-site interaction was significant only for CW and CS (Table 5). There were significantly positive genetic correlations 
between DBH, Volume, CW and CS as well as between HCB, BRA and SF (Table 7). In addition, it was indicated from Figures 5 and 6 that abilities of $\mathrm{DBH}, \mathrm{HCB}$, height, and $\mathrm{CW}$ to discriminate provenances and families were stronger than those of other traits. $\mathrm{DBH}, \mathrm{HCB}$, and height were thus used to estimate provenance-site interactions by biplot analysis for main genotypic effects and GGE based on the predicted breeding values, and CW was used to estimate the family-site interactions. In the bioplot of GGE, the cosine of the angle between the two environment segments indicated their correlations. Figure 1a showed the biplot of DBH for 25 provenances. There were sharp angles between segments of Changning, Hua'an and Pingxiang sites indicating positive correlations between them. Environment vector represented as an arrow vector joining center and co-ordinates of tested environment, its length indicated the discriminating ability on genotypic differences. Pingxiang showed the most discriminating ability for $\mathrm{DBH}$ in the present study. The biplot was divided into eight sectors in polygon view with provenances $\mathrm{U}, \mathrm{R}, \mathrm{P}, \mathrm{B}, \mathrm{L}, \mathrm{C}, \mathrm{A}$ and $\mathrm{S}$ as the vertexes. Vertex genotypes contributed overall most variation and demonstrated the best performance in each sector. From Figure 1a, it could be deduced that provenances L, B, and K were possible candidates for Mengla, and $\mathrm{R}$ and $\mathrm{V}$ for Pingxiang and Hua'an, and $\mathrm{P}$ for Changning based on DBH. In Figure $1 \mathrm{~b}$, the average environment axis (AEA) represented as an arrow vector joining the center and average coordinates of the tested site. Changning site was found to be the most representative environment for the growth of $\mathrm{DBH}$ as it has a smaller angle with AEA. The direction of AEA was the trend of the approximate mean for trait in all sites, it could be seen that provenance P showed the highest $\mathrm{DBH}$, followed by $\mathrm{W}, \mathrm{R}, \mathrm{V}$ and $\mathrm{Y}$, and the lowest DBH was observed in A and S. More variations in all four sites were observed in provenances $\mathrm{L}$ and $\mathrm{U}$ as it has longer perpendicular lines between their co-ordinates and AEX, and little variations for DBH were observed in provenances $\mathrm{I}, \mathrm{T}$ and $\mathrm{Y}$.

a

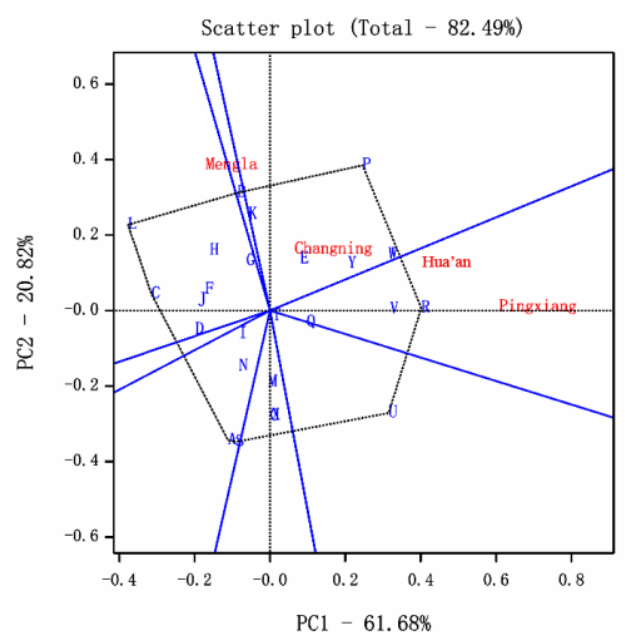

$\mathrm{b}$

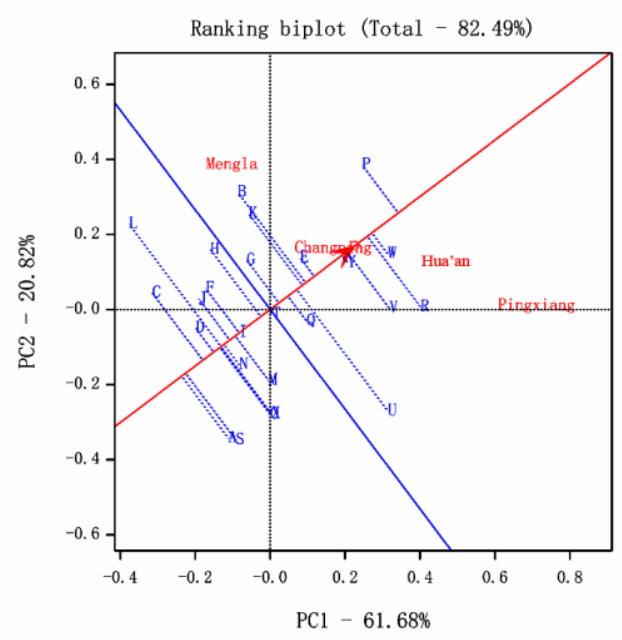

Figure 1. Biplot analysis based on diameter at breast height (DBH) data of 25 provenances. (a) Polygon view of genotype by environment (G×E) interaction (GGE) biplot-which is best for what, (b) genotypes of ranking provenances. A, Mengla; B, Yuanyang; C, Mojiang; D, Jinghong; E, Xichou; F, Zhenyuan; G, Tengchong; H, Jinggu; I, Ruili; J, Fengqing; K, Pingbian; L, Jiangcheng; M, Shuangjiang; N, Lancang; O, Lingyun; P, Longzhou; Q, Donglan; R, Tianlin; S, Debao; T, Tiane; U, Pingguo; V, Baise; W, Tianyang; $X$, Jingxi; $Y$, Napo.

Figure 2a showed the biplot for height, it was indicated that Changning and Hua'an had similar and higher environmental representativeness, and Pingxiang showed a higher ability to discriminate genotypic differences for tree height. Provenances L, C, and H were possible candidates for Mengla, and $\mathrm{P}$ for Changning and Hua'an, and V for Pingxiang based on height. Figure $2 \mathrm{~b}$ showed that height of provenance P was the highest, followed by G, Y, R, I and K, and the lowest height was observed in provenances $S$ and $X$. Little variations for height were observed in provenances $G$, I and P, and more variations were observed in $\mathrm{L}$ and $\mathrm{V}$. 
Figure $3 a$ showed the biplot of $\mathrm{HCB}$ for 25 provenances. Provenance $\mathrm{C}, \mathrm{L}$, and $\mathrm{F}$ were possible candidates for Mengla and Hua'an, and M, G, J and K for Changning and Pingxiang based on HCB. It was indicated from Figure $3 \mathrm{~b}$ that Changning and Hua' an had similar and higher environmental representativeness, and Mengla showed higher discriminating ability on genotypic differences. $\mathrm{HCB}$ of provenance $\mathrm{M}$ was the highest, followed by $\mathrm{C}, \mathrm{G}$, J, and $\mathrm{K}$, and the lowest HCB was observed in provenance $X$. Little variations for height were observed in provenances $N, S, L$ and $G$, and more variations in $\mathrm{C}$ and $\mathrm{Y}$.

a

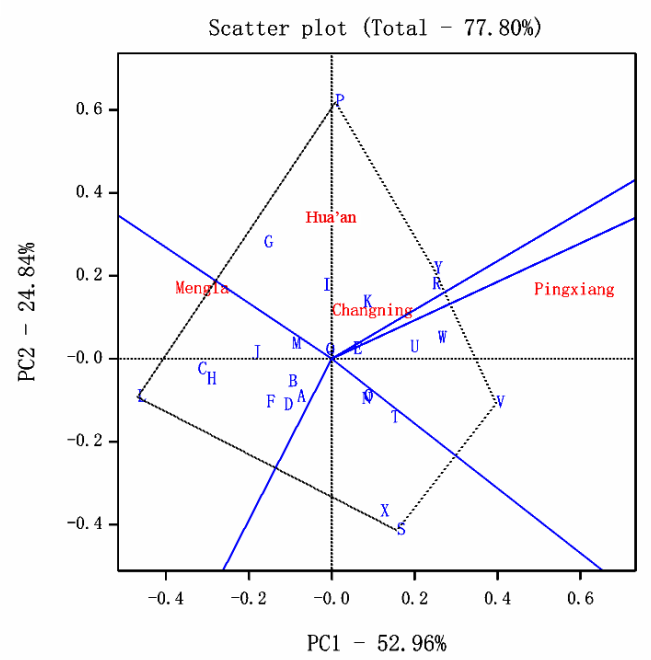

b

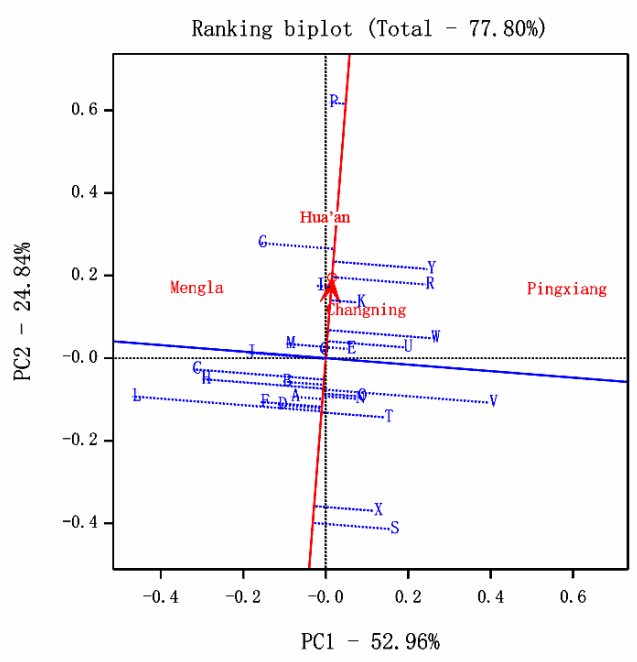

Figure 2. Biplot analysis based on the height of 25 provenances. (a) Polygon view of GGE biplot—which is best for what, (b) genotypes of ranking provenances. A, Mengla; B, Yuanyang; C, Mojiang; D, Jinghong; E, Xichou; F, Zhenyuan; G, Tengchong; H, Jinggu; I, Ruili; J, Fengqing; K, Pingbian; L, Jiangcheng; M, Shuangjiang; N, Lancang; O, Lingyun; P, Longzhou; Q, Donglan; R, Tianlin; S, Debao; T, Tiane; U, Pingguo; V, Baise; W, Tianyang; X, Jingxi; Y, Napo.

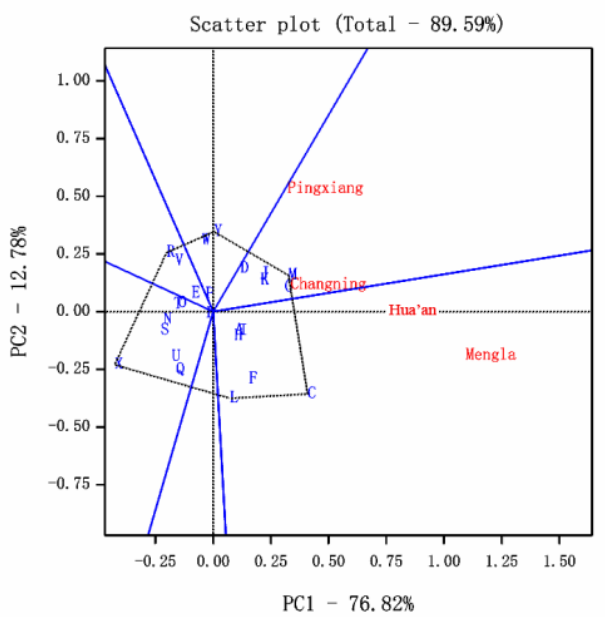

b

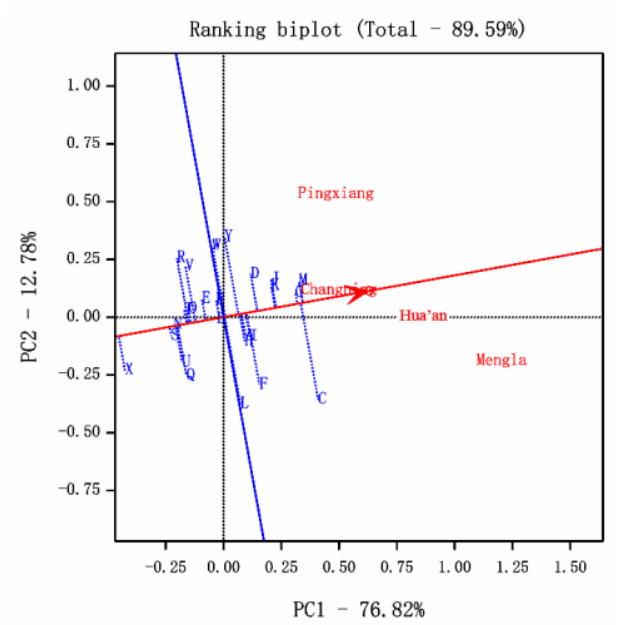

Figure 3. Biplot analysis based on HCB data of 25 provenances. (a) Polygon view of GGE biplot—which is best for what, (b) genotypes of ranking provenances. A, Mengla; B, Yuanyang; C, Mojiang; D, Jinghong; E, Xichou; F, Zhenyuan; G, Tengchong; H, Jinggu; I, Ruili; J, Fengqing; K, Pingbian; L, Jiangcheng; M, Shuangjiang; N, Lancang; O, Lingyun; P, Longzhou; Q, Donglan; R, Tianlin; S, Debao; T, Tiane; U, Pingguo; V, Baise; W, Tianyang; X, Jingxi; Y, Napo.

Figure 4 a showed the biplot of CW for 199 families. Families K08, P12, and W04 were possible candidates for Mengla, and R04, N20 and H18 for Pingxiang, Hua'an and Changning based on CW. From Figure $4 \mathrm{~b}$ it was indicated that Changning and Hua'an had similar and higher environmental 
representativeness, and Mengla and Pingxaing showed higher discriminating ability on genotypic differences. Family R04 was the highest, followed by N20, H18, and W04, and the lowest CW was observed in C03. Little variations for CW were observed in families C03, K07 and Q04, and more variations in Y01, L19 and A04.

a

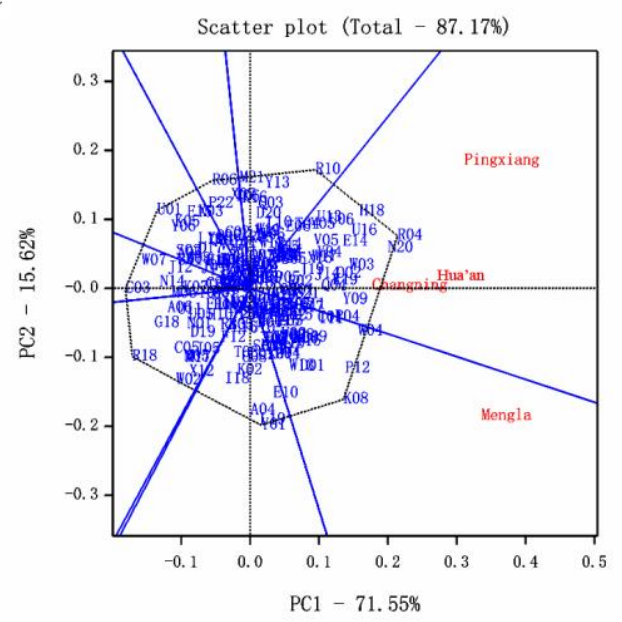

b

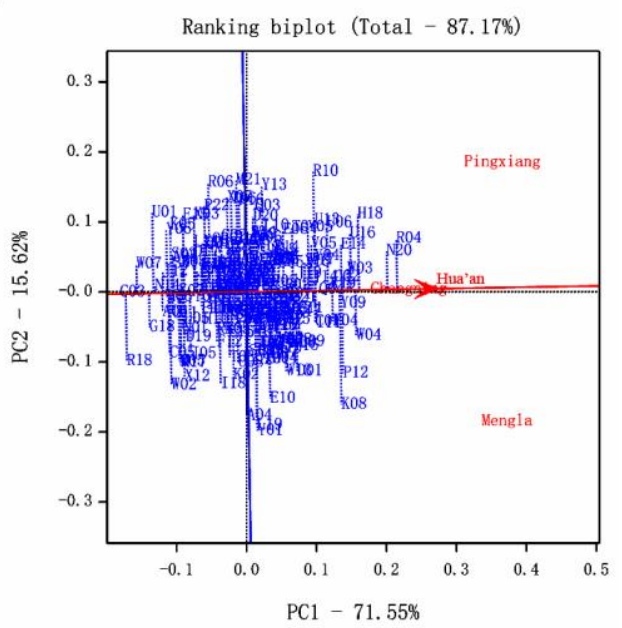

Figure 4. Biplot analysis based on CW data of 199 families. (a) Polygon view of GGE Biplot - which is best for what, (b) genotypes of ranking provenances. A, Mengla; B, Yuanyang; C, Mojiang; D, Jinghong; E, Xichou; F, Zhenyuan; G, Tengchong; H, Jinggu; I, Ruili; J, Fengqing; K, Pingbian; L, Jiangcheng; M, Shuangjiang; N, Lancang; O, Lingyun; P, Longzhou; Q, Donglan; R, Tianlin; S, Debao; T, Tiane; U, Pingguo; V, Baise; W, Tianyang; X, Jingxi; Y, Napo; Letters followed by numbers were the number of families.

\subsection{Superiority Rank and Selection for Provenances and Families}

The GGE biplot of which-won-where view at provenance level was presented in Figure 5a. The provenances were divided into seven sectors, $\mathrm{P}$ and $\mathrm{R}$ were possible candidates for $\mathrm{DBH}, \mathrm{CW}, \mathrm{CS}$, height and volume, and $\mathrm{G}$ and $\mathrm{K}$ were for $\mathrm{HCB}, \mathrm{BRA}$, and SF. In mean and stability view for trait performances of provenances (Figure $5 b$ ), DBH had the highest environmental representativeness, and height showed higher discriminating ability on genotypic differences. Provenances $\mathrm{H}, \mathrm{Y}$ and $\mathrm{L}$ had little variations for traits, and more variations were seen in provenances $\mathrm{X}, \mathrm{C}$, and $\mathrm{M}$. Additionally, provenance $\mathrm{P}$ performed the best for all traits, followed by $\mathrm{Y}$ and $\mathrm{W}$, the poorest performances were observed in $\mathrm{X}$ and $\mathrm{S}$. To rank the provenances based on their position in concentric circles, $\mathrm{P}$ was the most desirable provenance to be selected based on overall traits as it was located on the smallest concentric circle, follow by provenances $\mathrm{Y}, \mathrm{W}, \mathrm{K}$, and R.

Figure 6a showed the GGE biplot at family level, families P12, P04 and Y09 were possible candidates for DBH, height, Volume, CW, and CS, and H08, A06, and G11 were possible candidates for HCB, BRA, and SF. Ranking the families in Figure $6 \mathrm{~b}, \mathrm{P} 12$ was the most desirable family to be selected based on overall traits. Y09, W03, D01, and U06 were also desirable families. To rank the provenances and families based on their superiority and stability, excellent provenances and families were then screened out with a selection rate of $20 \%$ and $10 \%$, respectively. The means and realized gains of traits were presented in Table 8. It was inferred that not every trait could be positively selected. For example, the genetic and realized gains of HCB.height ${ }^{-1}$ were negative, while HCB, height, and crown width showed positive genetic and realized gains. These meant longer clear-wood and frondent crown, which were targets of selection in the present study. The genetic and realized gains of all traits except HCB.height ${ }^{-1}$ ranged from $0.25 \%$ to $2.01 \%$ and $2.43 \%$ to $14.84 \%$ at provenance level, and from $0.85 \%$ to $21.22 \%$ and $5.76 \%$ to $36.71 \%$ at family level, respectively. 


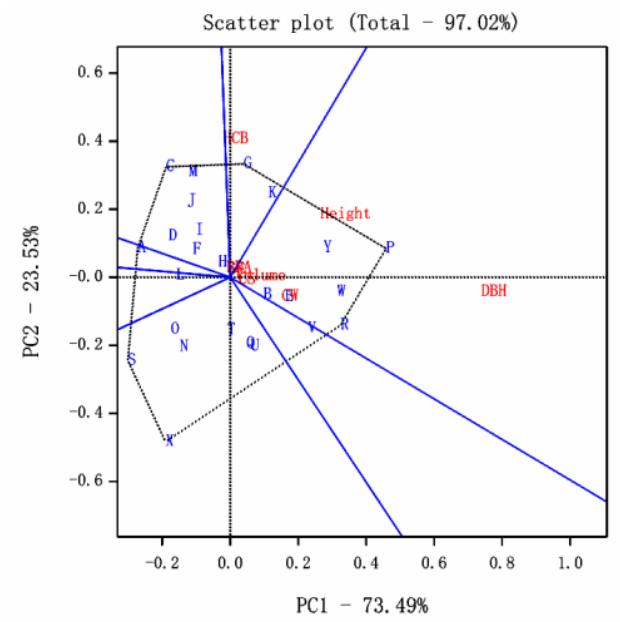

b

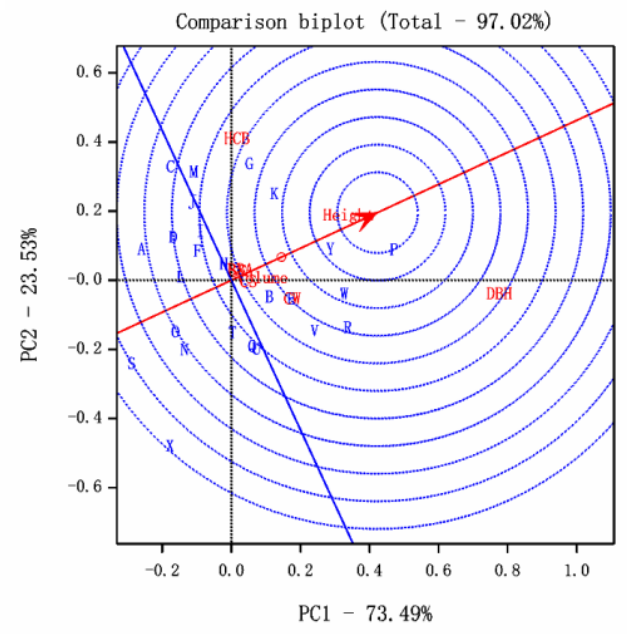

Figure 5. Biplot analysis based on all traits of 25 provenances. (a) Polygon view of GGE biplot-which is best for what, (b) genotypes of ranking provenances. $\mathrm{DBH}$, diameter at breast height; $\mathrm{HCB}$, height to live crown base; CW, crown width; SF, stem form; CS, crown shape; BRA, branchiness; A, Mengla; B, Yuanyang; C, Mojiang; D, Jinghong; E, Xichou; F, Zhenyuan; G, Tengchong; H, Jinggu; I, Ruili; J, Fengqing; K, Pingbian; L, Jiangcheng; M, Shuangjiang; N, Lancang; O, Lingyun; P, Longzhou; Q, Donglan; R, Tianlin; S, Debao; T, Tiane; U, Pingguo; V, Baise; W, Tianyang; X, Jingxi; Y, Napo.

a

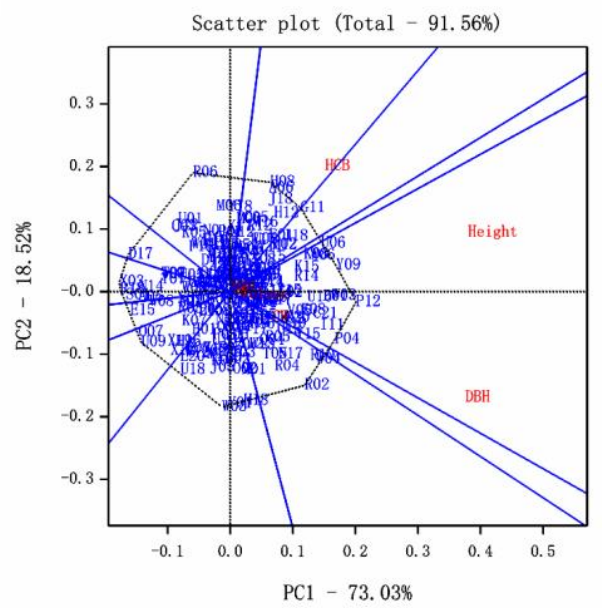

b

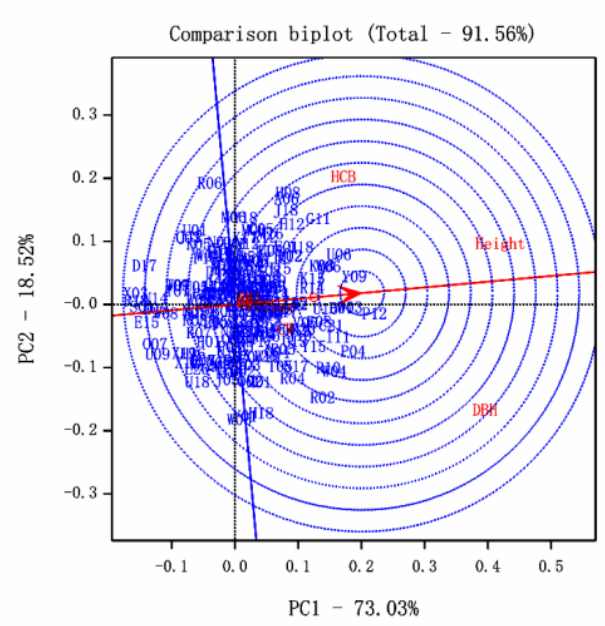

Figure 6. Biplot analysis based on all traits of 199 families. (a) Polygon view of GGE Biplot—which is best for what, (b) Genotypes of ranking families. DBH, diameter at breast height; HCB, height to live crown base; CW, crown width; SF, stem form; CS, crown shape; BRA, branchiness; A, Mengla; B, Yuanyang; C, Mojiang; D, Jinghong; E, Xichou; F, Zhenyuan; G, Tengchong; H, Jinggu; I, Ruili; J, Fengqing; K, Pingbian; L, Jiangcheng; M, Shuangjiang; N, Lancang; O, Lingyun; P, Longzhou; Q, Donglan; R, Tianlin; S, Debao; T, Tiane; U, Pingguo; V, Baise; W, Tianyang; X, Jingxi; Y, Napo; Letters followed by numbers were the number of families. 
Table 8. Growth, quality traits and realized gains of the excellent provenances and families.

\begin{tabular}{|c|c|c|c|c|c|c|c|c|c|c|c|c|c|c|}
\hline Provenances & $\begin{array}{l}\text { DBH } \\
\text { (cm) }\end{array}$ & $\begin{array}{c}\text { Height } \\
\text { (m) }\end{array}$ & $\begin{array}{l}\text { HCB } \\
(\mathrm{m})\end{array}$ & $\begin{array}{l}\mathrm{CW} \\
\text { (m) }\end{array}$ & $\begin{array}{l}\text { Volume } \\
\left(\mathrm{m}^{3}\right)\end{array}$ & $\mathrm{SF}$ & CS & BRA & $\begin{array}{c}\text { MADBH } \\
\left.\left(\mathrm{cm} \cdot Y^{-1}\right)^{-1}\right)\end{array}$ & $\underset{\left(m \cdot \text { Year }^{-1}\right)}{\text { MAH }}$ & HCB·Height ${ }^{-1}$ & $\begin{array}{l}\text { MAVOL } \\
\left(\mathrm{m}^{3} \cdot \text { Year }^{-1}\right)\end{array}$ & $\begin{array}{l}\text { Superiority } \\
\text { Coefficient }\end{array}$ & Rank \\
\hline$P$ & 18.58 & 16.12 & 8.42 & 3.91 & 0.27 & 0.66 & 0.71 & 0.69 & 1.33 & 1.16 & 0.53 & 0.02 & 0.0148 & 1 \\
\hline Y & 18.60 & 16.00 & 8.86 & 3.99 & 0.24 & 0.60 & 0.71 & 0.74 & 1.31 & 1.13 & 0.56 & 0.02 & 0.0269 & 2 \\
\hline $\mathrm{W}$ & 19.64 & 15.89 & 8.81 & 3.92 & 0.26 & 0.54 & 0.66 & 0.66 & 1.38 & 1.12 & 0.56 & 0.02 & 0.0378 & 3 \\
\hline $\mathrm{K}$ & 18.97 & 16.72 & 9.65 & 3.99 & 0.26 & 0.66 & 0.65 & 0.78 & 1.31 & 1.15 & 0.59 & 0.02 & 0.0457 & 4 \\
\hline $\mathrm{R}$ & 18.59 & 15.47 & 8.15 & 3.85 & 0.25 & 0.58 & 0.67 & 0.66 & 1.33 & 1.11 & 0.53 & 0.02 & 0.0578 & 5 \\
\hline Selected mean & 18.88 & 16.04 & 8.78 & 3.93 & 0.25 & 0.61 & 0.68 & 0.71 & 1.33 & 1.14 & 0.55 & 0.02 & & \\
\hline Total means & 17.59 & 15.34 & 8.56 & 3.78 & 0.22 & 0.62 & 0.66 & 0.72 & 1.25 & 1.09 & 0.56 & 0.02 & & \\
\hline Genetic gains (\%) & 2.01 & 0.30 & 1.55 & 0.90 & 0.39 & 1.59 & 0.42 & 0.80 & 1.88 & 0.25 & -1.01 & 0.38 & & \\
\hline Realized gains (\%) & 7.31 & 4.57 & 2.57 & 3.91 & 14.84 & 2.43 & 3.56 & 1.37 & 6.81 & 3.78 & -1.68 & 14.55 & & \\
\hline Families & $\begin{array}{l}\text { DBH } \\
(\mathrm{cm})\end{array}$ & $\begin{array}{c}\text { Height } \\
\text { (m) }\end{array}$ & $\begin{array}{c}\text { HCB } \\
(\mathrm{m})\end{array}$ & $\begin{array}{l}\mathrm{CW} \\
(\mathrm{m})\end{array}$ & $\begin{array}{c}\text { Volume } \\
\left(\mathrm{m}^{3}\right)\end{array}$ & SF & CS & BRA & $\begin{array}{c}\text { MADBH } \\
\left.\left(\mathrm{cm} \cdot Y^{-1}\right)^{-1}\right)\end{array}$ & $\begin{array}{c}\text { MAH } \\
\left(\mathrm{m} \cdot \text { Year }^{-1}\right)\end{array}$ & HCB·Height ${ }^{-1}$ & $\begin{array}{c}\text { MAVOL } \\
\left(\mathrm{m}^{3} \cdot \text { Year }^{-1}\right)\end{array}$ & $\begin{array}{l}\text { Superiority } \\
\text { Coefficient } \\
\end{array}$ & Rank \\
\hline P12 & 23.29 & 19.49 & 10.45 & 4.82 & 0.43 & 0.63 & 0.81 & 0.75 & 1.60 & 1.34 & 0.55 & 0.03 & 0.0092 & 1 \\
\hline Y09 & 19.49 & 17.33 & 9.72 & 4.63 & 0.31 & 0.64 & 0.84 & 0.85 & 1.39 & 1.24 & 0.57 & 0.02 & 0.0123 & 2 \\
\hline W03 & 21.88 & 18.37 & 9.88 & 4.90 & 0.35 & 0.67 & 0.82 & 0.81 & 1.50 & 1.25 & 0.55 & 0.02 & 0.0205 & 3 \\
\hline D01 & 18.48 & 15.48 & 9.68 & 4.20 & 0.22 & 0.77 & 0.80 & 0.92 & 1.32 & 1.13 & 0.58 & 0.01 & 0.0210 & 4 \\
\hline U06 & 20.46 & 18.89 & 10.86 & 5.00 & 0.33 & 0.66 & 0.76 & 0.72 & 1.39 & 1.28 & 0.58 & 0.02 & 0.0217 & 5 \\
\hline W06 & 19.33 & 16.59 & 9.74 & 3.85 & 0.29 & 0.56 & 0.59 & 0.70 & 1.37 & 1.19 & 0.58 & 0.02 & 0.0271 & 6 \\
\hline C21 & 22.69 & 19.37 & 11.69 & 4.78 & 0.41 & 0.84 & 0.86 & 0.89 & 1.53 & 1.31 & 0.61 & 0.03 & 0.0313 & 7 \\
\hline U16 & 19.11 & 16.14 & 8.87 & 4.33 & 0.26 & 0.60 & 0.74 & 0.73 & 1.36 & 1.16 & 0.55 & 0.02 & 0.0320 & 8 \\
\hline I11 & 22.24 & 19.78 & 10.06 & 5.00 & 0.37 & 0.88 & 0.82 & 0.82 & 1.50 & 1.34 & 0.51 & 0.03 & 0.0383 & 9 \\
\hline K08 & 20.68 & 18.81 & 10.53 & 4.78 & 0.32 & 0.80 & 0.75 & 0.68 & 1.41 & 1.28 & 0.57 & 0.02 & 0.0405 & 10 \\
\hline K15 & 21.81 & 18.00 & 11.54 & 4.09 & 0.33 & 0.49 & 0.70 & 0.77 & 1.48 & 1.22 & 0.64 & 0.02 & 0.0409 & 11 \\
\hline G11 & 20.22 & 19.08 & 11.49 & 4.33 & 0.31 & 0.68 & 0.79 & 0.84 & 1.39 & 1.32 & 0.61 & 0.02 & 0.0413 & 12 \\
\hline R14 & 19.28 & 16.52 & 9.02 & 4.00 & 0.26 & 0.49 & 0.65 & 0.76 & 1.37 & 1.18 & 0.56 & 0.02 & 0.0441 & 13 \\
\hline P04 & 22.03 & 18.95 & 8.92 & 4.59 & 0.41 & 0.77 & 0.75 & 0.65 & 1.52 & 1.31 & 0.50 & 0.03 & 0.0476 & 14 \\
\hline E08 & 20.81 & 17.56 & 9.20 & 4.22 & 0.29 & 0.63 & 0.78 & 0.66 & 1.44 & 1.21 & 0.55 & 0.02 & 0.0488 & 15 \\
\hline R10 & 22.37 & 17.72 & 8.85 & 4.63 & 0.35 & 0.72 & 0.69 & 0.69 & 1.55 & 1.22 & 0.50 & 0.02 & 0.0498 & 16 \\
\hline W04 & 23.50 & 18.21 & 8.82 & 4.69 & 0.38 & 0.57 & 0.72 & 0.65 & 1.64 & 1.27 & 0.48 & 0.03 & 0.0511 & 17 \\
\hline Y15 & 19.46 & 15.84 & 8.73 & 4.09 & 0.27 & 0.67 & 0.79 & 0.67 & 1.37 & 1.13 & 0.57 & 0.02 & 0.0516 & 18 \\
\hline V05 & 19.08 & 15.38 & 8.84 & 4.12 & 0.25 & 0.62 & 0.65 & 0.75 & 1.34 & 1.09 & 0.55 & 0.02 & 0.0527 & 19 \\
\hline I18 & 20.96 & 18.46 & 11.50 & 3.53 & 0.34 & 0.64 & 0.52 & 0.79 & 1.45 & 1.27 & 0.64 & 0.02 & 0.0533 & 20 \\
\hline Selected mean & 20.86 & 17.80 & 9.92 & 4.43 & 0.32 & 0.66 & 0.74 & 0.75 & 1.45 & 1.24 & 0.56 & 0.02 & & \\
\hline Total means & 18.34 & 15.88 & 8.89 & 3.89 & 0.24 & 0.61 & 0.66 & 0.71 & 1.28 & 1.11 & 0.57 & 0.02 & & \\
\hline Genetic gains (\%) & 5.87 & 5.17 & 5.73 & 5.21 & 21.22 & 1.46 & 1.92 & 0.85 & 5.43 & 4.67 & -0.37 & 20.92 & & \\
\hline Realized gains (\%) & 13.72 & 12.09 & 11.57 & 13.73 & 36.71 & 8.47 & 12.86 & 5.76 & 12.69 & 10.92 & -0.76 & 36.20 & & \\
\hline
\end{tabular}

Notes: The excellent provenances and families were selected with $20 \%$ and $10 \%$ selection rate, respectively. P, Daqingshan; Y, Napo; W, Tianyang; K, Pingbian; R, Tianlin; DBH, diameter at breast height; $\mathrm{HCB}$, height to live crown base; $\mathrm{CW}$, crown width; $\mathrm{SF}$, stem form; $\mathrm{CS}$, crown shape; $\mathrm{BRA}$, branchiness; MADBH, mean annual increment of diameter at breast height; $\mathrm{MAH}$ mean annual increment of tree height; HCB.Height ${ }^{-1}$, rate of height to live crown base to tree height; MAVOL, mean annual increment of stem volume. 


\section{Discussion}

Significant variances were observed for most growth and quality traits at levels of provenance and family, which showed good potential for elite germplasm selection of B. alnoides. The performances of growth traits were usually observed not consistent with those of quality traits for the same provenance. For example, the mean annual increment of diameter at breast height (MADBH), crown width (CW), height and volume were the lowest but stem form (SF) and branchiness (BRA) were the highest in provenance A. This discrepancy was also observed in other tree species like Pinus radiate [28], which make it difficult to take equally both growth and quality traits into consideration for selection of elite germplasms. A trade-off between growth and quality traits should thus be done according to the objective in the breeding program of $B$. alnoides.

Growth and quality traits of trees are mainly impacted by genotype, but also environment condition and physiological age [23]. Significant site effects were observed for all growth and quality traits tested except crown shape in the present study, indicating that B. alnoides trees had high phenotypic plasticity in these traits. Based on biplot analysis of GGE, Mengla and Pingxiang showed high discriminating ability of height, diameter at breast height (DBH), height to live crown base (HCB) and CW on genotypic differences, and similar environmental representativeness of Hua' an and Changning were observed in height, $\mathrm{DBH}, \mathrm{HCB}$ and $\mathrm{CW}$ traits. Site effects reflect the comprehensive reactions of trees to edaphic status, local and regional climatic conditions, etc. [29]. In the present study, the growth performance did not show an obvious trend with such a large range of altitude $(275-1250 \mathrm{~m})$, this is closely dependent on geomorphic features in southern China. But anyhow, mean annual air temperature and rainfall may play important roles in growth performance and showed less difference among four trial sites (Table 1). The relatively poor growth performance of B. alnoides at Changning site may partially be attributed to its shallow soil thickness and low rainfall compared to other sites.

The growth and quality traits varied among provenances and trial sites, which usually could be interpreted from the significant provenance-site interaction [30]. In the previous studies, there existed significant interactions between genotype and environment in tree height, DBH and stem volume for Larix kaempferi (Lamb.) Carriére [25] and in SF for Pinus radiate [28]. In the present study, significant provenance-site and provenance-block within site interactions were observed in most growth and quality traits based on combined variance analysis, demonstrating strong interactions between environment and provenance. While significant family-site interactions were seen only in CW and crown shape (CS). These inferred that the provenance-site interactions were more significant than family-site ones, this was consistent with the study on DBH and SF for Psuedotsuga menziesii (Mirb.) Franco [31].

$\mathrm{G} \times \mathrm{E}$ interaction will usually reduce the genetic gain by reducing the heritability at multi-site trials [12], while there was no significant decline in the heritability of most traits in the present study, which was a foundation for enough genetic gains obtained through selection. Selecting superior and stable for application at different sites was an efficient strategy for dealing with its significant $\mathrm{G} \times \mathrm{E}$ effects, and was also the main breeding program for B. alnoids. The tested traits showed low-to-moderate heritability in multi-site genetic analysis. The estimates of provenance repeatability for SF, BRA, and HCB were higher than those for other traits, this was in accordance with many previous studies such as the high provenance repeatability of stem straightness and branch traits for Pinus radiate [32]. While the estimates of family heritability for growth traits were higher than those for quality traits in the present study. Growth traits were also under more substantial genetic control than quality traits at family levels in the study of Pinus elliottii Engelm., which might be caused by a strong environment effect [23].

The phenotypic and genetic coefficient of variation (GCV, PCV) of volume and DBH were higher than those of height at each site, which indicated that volume and DBH were more sensitive to environmental influence than tree height. This was consistent with the studies on Populus deltoides Bartr. ex Marsh. [33]. The PCV of stem volume and quality traits were higher than other growth traits in both multi-site and single-site analysis, which indicated that $G \times E$ interaction of quality traits was 
stronger than that of most growth traits, and this was consistent with the above-mentioned result that growth traits were under stronger genetic control than quality traits. It was inferred that more genetic gains in terms of quality can be expected from selection at provenance level, and more genetic gains in terms of growth traits like volume can be expected from selection at family level for B. alnoides.

Significant correlations among traits indicated that growth and quality traits were not independent for B. alnoides. Moderate to strong positive genotypic correlations among DBH, height, and volume were observed. The correlations between DBH and volume were higher than those between height and volume, which showed the importance of DBH in selection for high stem volume [34]. The correlations between height with CW, SF and CS were not significant in the present study, while the previous study on clonal selection (3.5 years old unclosed forest) of Betula alnoides $\times$ Betula. luminifera H. Winkler hybrids showed that CW, SF, CS, and BRA were significantly correlated to height [21]. This difference could also be interpreted from the unstable growth performance and correlations between traits in the young plantations [20]. As stem volume and SF are the most concerned in plantation forestry of B. alnoides.

SF showed the highest while volume showed the lowest for provenance repeatability, indicating that genetic gain of SF was higher than that of volume in the selection for single trait. DBH, height, and HCB showed strong discriminating ability on provenance differences according to biplot analysis for GGE and were of moderate to strong genotypic correlations with most other traits. These inferred that it was possible to simplify the selected traits to DBH, HCB, and SF, and obtain good provenances with multi-trait. As to the family level, all growth traits had higher family heritabilities than quality traits. In particular, DBH, height, and HCB showed strong discriminating ability on family differences according to biplot analysis for GGE. In addition, moderate to strong cor relations usually existed between growth and quality traits. These inferred that it was possible to simplify the selected traits to volume, DBH, height, and HCB, and obtain good families with multi-trait.

As to methods for the analysis of $\mathrm{G} \times \mathrm{E}$ interaction and selection for excellent germplasms, variance analysis, principal coordinates analysis, and factor analysis as well as index selection and combined selection were widely used in the breeding process of trees [12,35,36]. However, these methods were usually applied to the analysis with a single site, population with simple genetic structure and non-visual form. The BLUP breeding value can be estimated for multi-trait in multi-site based on linear mixed models. Biplot analysis method can show the main genotypic effect $(G)$ and interaction effect $(\mathrm{G} \times \mathrm{E})$ of multi-site, and both superiority and stability of genotypes and the discriminating ability of traits can be visualized in biplot analysis for GGE according to the BLUP breeding value [26,36]. The present study also showed that the combination of BLUP breeding value and GGE biplot analysis is an appropriate method of multi-site selection for multiple traits.

For selection strategies in the present study, the desired provenances and families have been selected separately from provenances and families regardless of provenances. These germplasm can be applied in the practice. However, the trials were established with a single tree plot so as to evaluate more families. In the further study, multiple trees of a family will be arranged in each plot to reduce the environmental effect and improve the estimation of heritability and realized genetic gains, and these selected families and new families from the natural populations of the desired provenances will be involved in the progeny trials $[37,38]$. More superior families and individuals in families will thus be screened out for plantation forestry of B. alnoides.

\section{Conclusions}

Based on the combined analysis on growth and quality traits of 199 families of 25 provenances of B. alnoides at four sites when 10-15 years old, the genetic variance, heritability, correlation, and $\mathrm{G} \times \mathrm{E}$ interaction were estimated and the provenances and families were ranked. The results showed that there existed a remarkable site, provenance, family, and provenance-site effects for tested traits, indicating the significance of $\mathrm{G} \times \mathrm{E}$ interaction. The provenance repeatability of $\mathrm{HCB}, \mathrm{SF}$, and BRA showed higher levels than that of other traits. Growth traits were under moderate and relatively higher 
genetic control rather than quality traits based on family heritabilities. The $\mathrm{DBH}$, height, $\mathrm{HCB}$, and $\mathrm{CW}$ were under relatively higher genetic control, had a stronger discriminating ability on genotype differences, and were also strongly correlated with other traits, they should be paid more attention to in the multi-trait selection. The superior and stable provenances and families selected by GGE biplot analysis should be applied in the practice of $B$. alnoides plantation forestry.

Author Contributions: J.Z., J.G., C.W., and Z.Z. designed the experiment; M.Y., J.G., C.W., Z.Z. and J.Z. performed the experiments and collected the data; M.Y. and J.G. analyzed the data; M.Y. and J.G. contributed to writing the manuscript.

Funding: This work was supported by the National Nonprofit Institute Research Grant of Chinese Academy of Forestry, China (CAFYBB2017SY019), Forestry Science and Technology Innovation Project of Guangdong, China (2017KJCX032) and the National Key Research and Development Program of China (2016YFD0600604).

Acknowledgments: We would like to thank Experimental Center of Tropical Forestry, Chinese Academy of Forestry (ECTF), Baoshan Forest Extension Station (BFES), Mengla County Forestry and Grassland Bureau (MCFGB) and Jinshan National Forest Farm of Hua'an (JNFFH) for their permission to establish trial plantations, and thank Wenfu Guo in ECTF, Yanping Yang and Jiacong Huang in BFES, Xiancheng Zhu in MCFGB and Bihua Chen in Fujian Academy of Forestry Sciences for their assistance in management and growth measurement of the trial plantations. We were also grateful to the editor and anonymous reviewers for their careful revisions and constructive comments.

Conflicts of Interest: We declare that we have no conflict of interest.

\section{Appendix A}

Table A1. Location of 25 provenances of Betula alnoids investigated in the trials.

\begin{tabular}{|c|c|c|c|c|c|}
\hline Provenances & Location & Latitude $\mathbf{N}$ & Longitude E & Altitude (m) & Number of Family Tested \\
\hline $\mathrm{A}$ & Mengla, Yunnan & $21^{\circ} 33^{\prime}$ & $101^{\circ} 29^{\prime}$ & $950-1100$ & 3 \\
\hline $\mathrm{B}$ & Yuanyang, Yunnan & $23^{\circ} 07^{\prime}$ & $103^{\circ} 01^{\prime}$ & $1000-1130$ & 3 \\
\hline $\mathrm{C}$ & Mojiang, Yunnan & $23^{\circ} 30^{\prime}$ & $101^{\circ} 46^{\prime}$ & $1500-1600$ & 12 \\
\hline $\mathrm{D}$ & Jinghong, Yunnan & $22^{\circ} 24^{\prime}$ & $100^{\circ} 59^{\prime}$ & 1300 & 7 \\
\hline $\mathrm{E}$ & Xichou, Yunnan & $23^{\circ} 15^{\prime}$ & $104^{\circ} 33^{\prime}$ & $1400-1600$ & 8 \\
\hline $\mathrm{F}$ & Zhenyuan, Yunnan & $23^{\circ} 59^{\prime}$ & $101^{\circ} 09^{\prime}$ & $1570-1900$ & 4 \\
\hline G & Tengchong, Yunnan & $24^{\circ} 56^{\prime}$ & $98^{\circ} 36^{\prime}$ & $1820-2100$ & 2 \\
\hline $\mathrm{H}$ & Jingguo, Yunnan & $23^{\circ} 29^{\prime}$ & $100^{\circ} 34^{\prime}$ & $1650-1800$ & 9 \\
\hline $\mathrm{I}$ & Ruili, Yunnan & $23^{\circ} 56^{\prime}$ & $98^{\circ} 03^{\prime}$ & $1120-1250$ & 8 \\
\hline $\mathrm{J}$ & Fengqing, Yunnan & $24^{\circ} 54^{\prime}$ & $99^{\circ} 55^{\prime}$ & $1380-1850$ & 12 \\
\hline $\mathrm{K}$ & Pingbian, Yunnan & $23^{\circ} 03^{\prime}$ & $103^{\circ} 38^{\prime}$ & $1230-1320$ & 10 \\
\hline $\mathrm{L}$ & Jiangcheng, Yunnan & $22^{\circ} 35^{\prime}$ & $101^{\circ} 47^{\prime}$ & $1190-1280$ & 10 \\
\hline M & Shuangjiang, Yunnan & $23^{\circ} 26^{\prime}$ & $99^{\circ} 39^{\prime}$ & $1560-1670$ & 7 \\
\hline $\mathrm{N}$ & Lancang, Yunnan & $22^{\circ} 36^{\prime}$ & $99^{\circ} 59^{\prime}$ & $1280-1500$ & 9 \\
\hline $\mathrm{O}$ & Lingyun, Gaungxi & $24^{\circ} 00^{\prime}$ & $106^{\circ} 35^{\prime}$ & $700-900$ & 6 \\
\hline $\mathrm{P}$ & Longzhou, Gaungxi & $22^{\circ} 25^{\prime}$ & $106^{\circ} 40^{\prime}$ & $600-800$ & 8 \\
\hline Q & Donglan, Gaungxi & $24^{\circ} 29^{\prime}$ & $107^{\circ} 34^{\prime}$ & $500-680$ & 3 \\
\hline$\hat{\mathrm{R}}$ & Tianlin, Gaungxi & $24^{\circ} 25^{\prime}$ & $105^{\circ} 43^{\prime}$ & $740-920$ & 11 \\
\hline $\mathrm{S}$ & Debao, Gaungxi & $23^{\circ} 20^{\prime}$ & $106^{\circ} 38^{\prime}$ & $800-900$ & 4 \\
\hline $\mathrm{T}$ & Tian'e, Gaungxi & $25^{\circ} 11^{\prime}$ & $107^{\circ} 13^{\prime}$ & $650-750$ & 7 \\
\hline $\mathrm{U}$ & Pingguo, Gaungxi & $23^{\circ} 42^{\prime}$ & $107^{\circ} 23^{\prime}$ & $650-800$ & 12 \\
\hline $\mathrm{V}$ & Baise, Gaungxi & $23^{\circ} 47^{\prime}$ & $106^{\circ} 30^{\prime}$ & $500-900$ & 5 \\
\hline W & Tianyang, Gaungxi & $23^{\circ} 52^{\prime}$ & $107^{\circ} 08^{\prime}$ & $550-700$ & 15 \\
\hline$x$ & Jingxi, Gaungxi & $23^{\circ} 01^{\prime}$ & $106^{\circ} 20^{\prime}$ & $800-1000$ & 10 \\
\hline $\mathrm{Y}$ & Napo, Gaungxi & $23^{\circ} 11^{\prime}$ & $105^{\circ} 51^{\prime}$ & $600-850$ & 14 \\
\hline
\end{tabular}


Table A2. Growth and quality traits for 25 provenances of Betula alnoids at Mengla site.

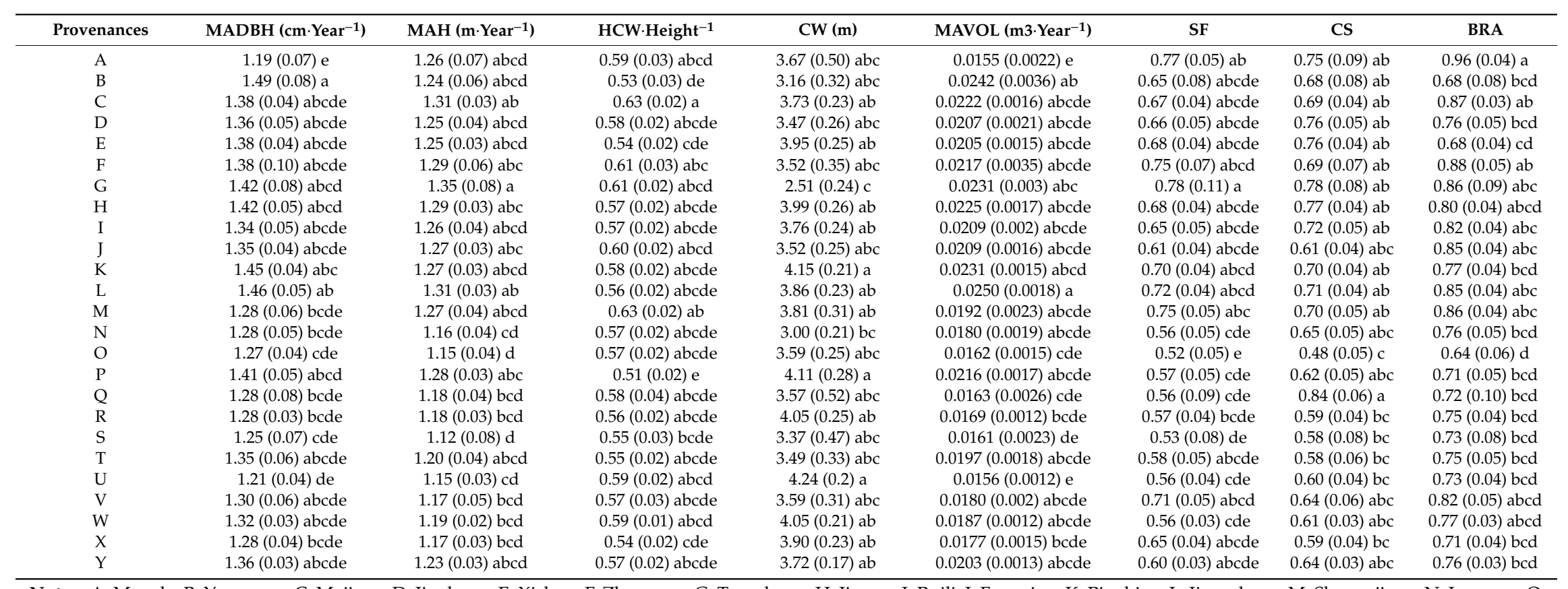

Notes: A, Mengla; B, Yuanyang; C, Mojiang; D, Jinghong; E, Xichou; F, Zhenyuan; G, Tengchong; H, Jinggu; I, Ruili; J, Fengqing; K, Pingbian; L, Jiangcheng; M, Shuangjiang; N, Lancang; O, Lingyun; P, Longzhou; Q, Donglan; R, Tianlin; S, Debao; T, Tiane; U, Pingguo; V, Baise; W, Tianyang; X, Jingxi; Y, Napo; MADBH, mean annual increment of diameter at breast height; $\mathrm{MAH}$, mean annual increment of tree height; HCB.Height ${ }^{-1}$, rate of height to live crown base to tree height; CW, crown width; MAVOL, mean annual increment of stem volume; SF, stem wholly different small letters in the same row. 
Table A3. Growth and quality traits for 25 provenances of Betula alnoids at Pingxiang site.

\begin{tabular}{|c|c|c|c|c|c|c|c|c|}
\hline Provenances & MADBH (cm·Year-1) & MAH $\left(m \cdot\right.$ Year $\left.^{-1}\right)$ & HCW·Height ${ }^{-1}$ & $\mathrm{CW}(\mathrm{m})$ & MAVOL (m3·Year-1) & SF & CS & BRA \\
\hline $\mathrm{A}$ & $0.89(0.05)$ fghi & $0.85(0.06)$ bcdef & $0.67(0.05)$ abcde & $5.59(0.76) \mathrm{ab}$ & $0.0058(0.0009)$ def & $0.74(0.07)$ & 0.59 (0.14) abcd & $0.74(0.13)$ \\
\hline B & $0.94(0.09)$ defghi & $0.85(0.06)$ bcdef & $0.64(0.02)$ abcdef & $4.36(0.39) b c$ & 0.0075 (0.0021) bcdef & $0.79(0.08)$ & $0.68(0.06)$ abcd & $0.77(0.09)$ \\
\hline C & 0.84 (0.04) fghi & $0.83(0.03)$ cdef & $0.66(0.02)$ abcd & $3.90(0.34) \mathrm{cd}$ & $0.0058(0.0008)$ cdef & $0.58(0.06)$ & $0.55(0.06) \mathrm{bcd}$ & $0.69(0.06)$ \\
\hline $\mathrm{D}$ & $0.91(0.06)$ efghi & $0.87(0.04)$ bcdef & $0.70(0.02) \mathrm{a}$ & $3.05(0.31) \mathrm{d}$ & 0.0067 (0.0012) cdef & $0.73(0.06)$ & $0.66(0.05) \mathrm{bcd}$ & $0.88(0.05)$ \\
\hline E & 1.11 (0.04) abcdef & $0.97(0.05)$ abcde & $0.59(0.02)$ cdef & $4.37(0.31) \mathrm{bcd}$ & 0.0105 (0.0011) abcdef & $0.69(0.06)$ & $0.67(0.05) \mathrm{abcd}$ & $0.75(0.05)$ \\
\hline $\mathrm{F}$ & $0.79(0.09) \mathrm{hi}$ & $0.81(0.08)$ def & $0.59(0.04)$ def & $3.59(0.35) \mathrm{cd}$ & 0.0055 (0.0018) ef & $0.70(0.07)$ & $0.57(0.12) \mathrm{bcd}$ & $0.77(0.07)$ \\
\hline G & 0.87 (0.07) ghi & $0.87(0.07)$ bcdef & $0.69(0.04) \mathrm{ab}$ & $3.36(0.52) \mathrm{cd}$ & 0.0059 (0.0014) cdef & $0.66(0.13)$ & $0.51(0.15) \mathrm{cd}$ & $0.85(0.08)$ \\
\hline $\mathrm{H}$ & $0.95(0.08)$ cdefghi & $0.80(0.05)$ ef & $0.69(0.02) \mathrm{ab}$ & $3.63(0.46) \mathrm{cd}$ & $0.0080(0.0020)$ bcdef & $0.75(0.06)$ & $0.48(0.06) \mathrm{d}$ & $0.71(0.07)$ \\
\hline I & $0.90(0.07)$ bcdefghi & $0.96(0.06)$ abcde & $0.62(0.03)$ abcdef & $3.73(0.59) \mathrm{cd}$ & $0.0088(0.0018)$ bcdef & $0.63(0.07)$ & $0.52(0.07) \mathrm{bcd}$ & $0.62(0.09)$ \\
\hline $\mathrm{J}$ & $0.83(0.04) \mathrm{hi}$ & $0.86(0.04)$ bcdef & $0.69(0.02) \mathrm{a}$ & $3.49(0.34) \mathrm{cd}$ & 0.0054 (0.0007) ef & $0.64(0.07)$ & $0.46(0.07) \mathrm{d}$ & $0.78(0.06)$ \\
\hline $\mathrm{K}$ & $1.06(0.04)$ abcdefg & $1.00(0.04) \mathrm{abc}$ & $0.60(0.01)$ cdef & $3.11(0.27) \mathrm{cd}$ & 0.0106 (0.0012) abcde & $0.63(0.05)$ & $0.64(0.05) \mathrm{abcd}$ & $0.83(0.05)$ \\
\hline $\mathrm{L}$ & $0.81(0.05) \mathrm{i}$ & $0.74(0.03) \mathrm{f}$ & $0.65(0.03)$ abcde & $3.35(0.35) \mathrm{cd}$ & $0.0047(0.0009) \mathrm{f}$ & $0.74(0.05)$ & $0.47(0.07) \mathrm{d}$ & $0.71(0.07)$ \\
\hline M & $1.01(0.05)$ bcdefghi & $0.91(0.05)$ abcde & $0.68(0.02) \mathrm{abc}$ & $4.00(0.54) \mathrm{bcd}$ & $0.0084(0.0012)$ bcdef & $0.75(0.07)$ & $0.42(0.07) \mathrm{d}$ & $0.80(0.09)$ \\
\hline $\mathrm{N}$ & $0.89(0.04)$ fghi & $0.89(0.04)$ bcdef & $0.61(0.03)$ abcdef & $2.91(0.33) \mathrm{d}$ & $0.0062(0.0008)$ cdef & $0.69(0.07)$ & $0.51(0.06) \mathrm{bcd}$ & $0.75(0.06)$ \\
\hline $\mathrm{O}$ & $1.07(0.04)$ abcdefg & $0.89(0.04)$ bcdef & $0.64(0.02)$ abcde & $3.43(0.36) \mathrm{cd}$ & $0.0086(0.0010)$ bcdef & $0.77(0.07)$ & $0.60(0.08) \mathrm{bcd}$ & $0.78(0.06)$ \\
\hline $\mathrm{P}$ & $1.12(0.07)$ abcd & $0.99(0.05)$ abcd & $0.59(0.02)$ def & $3.36(0.32) \mathrm{cd}$ & $0.0130(0.0018) \mathrm{ab}$ & $0.76(0.04)$ & $0.71(0.06) \mathrm{abcd}$ & $0.81(0.05)$ \\
\hline $\mathrm{Q}$ & 1.05 (0.05) abcdefghi & $0.89(0.03)$ bcdef & $0.57(0.03)$ def & $3.47(0.37) \mathrm{cd}$ & 0.0084 (0.0011) bcdef & $0.66(0.06)$ & $0.75(0.08) \mathrm{abc}$ & $0.64(0.10)$ \\
\hline $\mathrm{R}$ & $1.21(0.05) \mathrm{ab}$ & $1.00(0.04) \mathrm{abc}$ & $0.60(0.02)$ cdef & $3.57(0.26) \mathrm{cd}$ & $0.0133(0.0012) \mathrm{ab}$ & $0.69(0.05)$ & $0.64(0.06) \mathrm{bcd}$ & $0.74(0.04)$ \\
\hline $\mathrm{s}$ & $1.06(0.07)$ abcdefg & $0.94(0.05)$ abcde & $0.56(0.05) \mathrm{f}$ & $6.18(0.53)$ a & $0.0092(0.0015)$ bcdef & $0.70(0.08)$ & $0.89(0.06) \mathrm{a}$ & $0.65(0.13)$ \\
\hline $\mathrm{T}$ & $1.08(0.05)$ abcdefg & 0.98 (0.04) abcde & $0.57(0.02) \mathrm{def}$ & $4.22(0.31) \mathrm{bcd}$ & $0.0103(0.0015)$ abcdef & $0.71(0.06)$ & $0.76(0.06) \mathrm{abc}$ & $0.81(0.05)$ \\
\hline $\mathrm{U}$ & $1.14(0.04)$ abcde & 0.95 (0.03) abcde & $0.56(0.01)$ ef & $3.93(0.23) \mathrm{cd}$ & 0.0115 (0.0011) abcd & $0.70(0.04)$ & $0.71(0.04) \mathrm{abcd}$ & $0.64(0.04)$ \\
\hline $\mathrm{V}$ & $1.27(0.06) \mathrm{a}$ & $1.12(0.05) \mathrm{a}$ & $0.56(0.02)$ ef & $4.00(0.33) \mathrm{cd}$ & $0.0152(0.0020) \mathrm{a}$ & $0.71(0.07)$ & $0.77(0.06) \mathrm{abc}$ & $0.82(0.05)$ \\
\hline $\mathrm{W}$ & $1.14(0.04)$ abcde & $1.00(0.03) a b c$ & $0.60(0.01)$ bcdef & $3.15(0.22) \mathrm{cd}$ & 0.0117 (0.0011) abc & $0.70(0.04)$ & $0.62(0.04) \mathrm{bcd}$ & $0.75(0.04)$ \\
\hline $\mathrm{x}$ & $1.05(0.05)$ abcdefgh & $0.92(0.04)$ bcde & $0.59(0.02) \mathrm{def}$ & $4.32(0.28) \mathrm{bcd}$ & 0.0098 (0.0011) abcdef & $0.70(0.05)$ & $0.77(0.04) \mathrm{ab}$ & $0.71(0.05)$ \\
\hline $\mathrm{Y}$ & $1.17(0.04) \mathrm{abc}$ & $1.03(0.03) \mathrm{ab}$ & $0.58(0.01) \mathrm{def}$ & $3.83(0.23) \mathrm{cd}$ & $0.0129(0.0011) \mathrm{ab}$ & $0.63(0.04)$ & $0.77(0.04) \mathrm{ab}$ & $0.79(0.04)$ \\
\hline
\end{tabular}

Notes: A, Mengla; B, Yuanyang; C, Mojiang; D, Jinghong; E, Xichou; F, Zhenyuan; G, Tengchong; H, Jinggu; I, Ruili; J, Fengqing; K, Pingbian; L, Jiangcheng; M, Shuangiiang; N, Lancang; O, Lingyun; P, Longzhou; Q, Donglan; R, Tianlin; S, Debao; T, Tiane; U, Pingguo; V, Baise; W, Tianyang; X, Jingxi; Y, Napo; MADBH, mean annual increment of diameter at breast height; $\mathrm{MAH}$, mean annual increment of tree height; HCB.Height ${ }^{-1}$, rate of height to live crown base to tree height; CW, crown width; MAVOL, mean annual increment of stem volume; SF, stem form; CS, crown shape; BRA, branchiness. Means with standard error in parenthesis were of significant difference at 0.05 level according to Duncan's multiple range tests if followed by wholly different small letters in the same row. 
Table A4. Growth and quality traits for 25 provenances of Betula alnoids at Hua'an site.

\begin{tabular}{|c|c|c|c|c|c|c|c|c|}
\hline Provenances & MADBH $\left(\mathrm{cm} \cdot\right.$ Year $\left.^{-1}\right)$ & MAH $\left(m \cdot\right.$ Year $\left.^{-1}\right)$ & HCW.Height ${ }^{-1}$ & $\mathrm{CW}(\mathrm{m})$ & MAVOL $\left(m^{3} \cdot\right.$ Year $\left.^{-1}\right)$ & $\mathrm{SF}$ & CS & BRA \\
\hline A & $1.31(0.09) \mathrm{e}$ & $1.14(0.09) \mathrm{cd}$ & $0.62(0.05) \mathrm{ab}$ & $4.03(0.47) \mathrm{abcd}$ & $0.0135(0.0023) \mathrm{e}$ & $0.70(0.15) \mathrm{ab}$ & $0.36(0.11) \mathrm{h}$ & 0.74 (0.14) abcde \\
\hline B & $1.49(0.07)$ abcde & $1.17(0.05) \mathrm{abcd}$ & $0.60(0.02) a b c$ & $3.17(0.36) \mathrm{de}$ & $0.0190(0.0022)$ bcde & $0.57(0.08) \mathrm{abc}$ & $0.65(0.07)$ bcdef & $0.73(0.07)$ abcde \\
\hline C & $1.38(0.06)$ bcde & $1.15(0.04) \mathrm{bcd}$ & $0.60(0.02) \mathrm{abcd}$ & $3.61(0.27) \mathrm{bcd}$ & $0.0172(0.0021)$ bcde & $0.49(0.07) \mathrm{cd}$ & $0.56(0.06)$ cdefgh & $0.70(0.05)$ abcde \\
\hline $\mathrm{D}$ & $1.37(0.07)$ cde & $1.14(0.04) \mathrm{cd}$ & $0.60(0.02) \mathrm{abcd}$ & $4.50(0.28) \mathrm{ab}$ & $0.0161(0.0019) \mathrm{de}$ & $0.64(0.09) \mathrm{abc}$ & $0.46(0.07) \mathrm{fgh}$ & $0.73(0.06)$ abcde \\
\hline $\mathrm{E}$ & $1.47(0.04)$ abcde & $1.15(0.03) \mathrm{cd}$ & $0.56(0.02)$ abcde & $4.14(0.23) a b c$ & $0.0190(0.0012)$ bcde & $0.45(0.04) \mathrm{cd}$ & $0.72(0.04) \mathrm{abcd}$ & $0.58(0.04)$ cde \\
\hline $\mathrm{F}$ & $1.46(0.10)$ abcde & $1.12(0.03) \mathrm{cd}$ & $0.66(0.02) \mathrm{a}$ & $3.54(0.51) \mathrm{bcd}$ & $0.0179(0.0027)$ bcde & $0.64(0.07) \mathrm{abc}$ & $0.40(0.08) \mathrm{gh}$ & $0.89(0.05) \mathrm{a}$ \\
\hline G & $1.58(0.09) \mathrm{ab}$ & $1.36(0.07) \mathrm{a}$ & $0.59(0.04)$ abcde & $4.83(0.42) \mathrm{a}$ & $0.0242(0.0036)$ abcd & $0.57(0.09) \mathrm{abc}$ & $0.77(0.1) \mathrm{abc}$ & $0.73(0.09)$ abcde \\
\hline $\mathrm{H}$ & $1.41(0.05)$ bcde & $1.17(0.03) \mathrm{abcd}$ & $0.55(0.03)$ bcdef & $3.50(0.28) \mathrm{bcd}$ & $0.0172(0.0015)$ bcde & $0.58(0.07) \mathrm{abc}$ & $0.61(0.07)$ bcdefg & $0.75(0.05)$ abcd \\
\hline I & $1.56(0.07) \mathrm{abc}$ & $1.25(0.04) \mathrm{abc}$ & $0.58(0.03)$ abcde & $4.36(0.41) \mathrm{abc}$ & $0.0225(0.0026) \mathrm{abcd}$ & $0.65(0.08) a b c$ & $0.54(0.09)$ cdefgh & $0.70(0.07)$ abcde \\
\hline $\mathrm{J}$ & $1.52(0.04)$ abcde & $1.18(0.03) \mathrm{abcd}$ & $0.59(0.02)$ abcde & $3.93(0.27) \mathrm{abcd}$ & $0.0201(0.0015) \mathrm{bcde}$ & $0.58(0.05) \mathrm{abc}$ & $0.55(0.06)$ cdefgh & $0.67(0.05)$ abcde \\
\hline $\mathrm{K}$ & $1.41(0.04)$ bcde & $1.16(0.03) \mathrm{abcd}$ & $0.59(0.02)$ abcde & $3.90(0.27)$ abcd & $0.0174(0.0013)$ bcde & $0.56(0.06) \mathrm{abc}$ & $0.58(0.06)$ cdefgh & $0.77(0.04) a b c$ \\
\hline $\mathrm{L}$ & $1.34(0.07) \mathrm{de}$ & $1.17(0.05) \mathrm{abcd}$ & $0.60(0.03)$ abcde & $3.48(0.33) \mathrm{bcd}$ & $0.0168(0.0023) \mathrm{cde}$ & $0.74(0.06) \mathrm{a}$ & $0.47(0.07)$ efgh & $0.78(0.07) \mathrm{ab}$ \\
\hline M & $1.49(0.06)$ abcde & $1.19(0.04) \mathrm{abcd}$ & $0.59(0.03)$ abcde & $2.41(0.23) \mathrm{e}$ & $0.0193(0.0018)$ bcde & $0.58(0.07) \mathrm{abc}$ & $0.47(0.07) \mathrm{fgh}$ & $0.74(0.07)$ abcde \\
\hline $\mathrm{N}$ & $1.54(0.10) \mathrm{abcd}$ & $1.20(0.05) \mathrm{abcd}$ & $0.57(0.03)$ abcde & $3.42(0.3) \mathrm{cde}$ & $0.0244(0.0041) \mathrm{abc}$ & $0.44(0.08) \mathrm{cd}$ & $0.51(0.08)$ defgh & $0.57(0.07)$ cde \\
\hline $\mathrm{O}$ & $1.41(0.04)$ bcde & $1.22(0.04) \mathrm{abc}$ & $0.54(0.02)$ cdef & $3.31(0.23) \mathrm{cde}$ & $0.0176(0.0013)$ bcde & $0.54(0.05) \mathrm{bcd}$ & $0.73(0.05) \mathrm{abcd}$ & $0.64(0.05)$ bcde \\
\hline $\mathrm{P}$ & $1.64(0.06) \mathrm{a}$ & $1.31(0.04) \mathrm{ab}$ & $0.54(0.02) \mathrm{cdef}$ & $3.85(0.23) \mathrm{abcd}$ & $0.0278(0.0025)$ a & $0.62(0.05) \mathrm{abc}$ & $0.81(0.05) \mathrm{ab}$ & $0.62(0.05)$ bcde \\
\hline $\mathrm{Q}$ & $1.59(0.05) \mathrm{ab}$ & $1.22(0.04) a b c$ & $0.48(0.03) \mathrm{f}$ & $3.38(0.33) \mathrm{cde}$ & $0.0216(0.0016)$ abcd & $0.52(0.07) \mathrm{bcd}$ & $0.92(0.04) \mathrm{a}$ & $0.60(0.07) \mathrm{cde}$ \\
\hline $\mathrm{R}$ & $1.61(0.04) \mathrm{ab}$ & $1.21(0.03) \mathrm{abcd}$ & $0.50(0.01)$ ef & $3.64(0.16) \mathrm{bcd}$ & $0.0256(0.0017) \mathrm{ab}$ & $0.46(0.03) \mathrm{cd}$ & $0.71(0.03) \mathrm{abcd}$ & $0.53(0.03) \mathrm{e}$ \\
\hline $\mathrm{s}$ & $1.30(0.06) \mathrm{e}$ & $1.06(0.04) \mathrm{d}$ & $0.56(0.03)$ abcdef & $3.95(0.33) \mathrm{abcd}$ & $0.0132(0.0015) \mathrm{e}$ & $0.33(0.07) \mathrm{d}$ & $0.60(0.07)$ cdefg & $0.71(0.07)$ abcde \\
\hline $\mathrm{T}$ & $1.42(0.04)$ abcde & $1.13(0.03) \mathrm{cd}$ & $0.56(0.02)$ bcdef & $3.77(0.19)$ abcd & $0.0166(0.0012)$ cde & $0.42(0.05) \mathrm{cd}$ & $0.66(0.05)$ bcdef & $0.60(0.05)$ bcde \\
\hline $\mathrm{U}$ & $1.61(0.05) \mathrm{ab}$ & $1.22(0.04) \mathrm{abc}$ & $0.52(0.02)$ def & $3.84(0.22)$ abcd & $0.0246(0.0018) \mathrm{ab}$ & $0.43(0.04) \mathrm{cd}$ & $0.69(0.04)$ abcde & $0.54(0.04) \mathrm{de}$ \\
\hline $\mathrm{V}$ & $1.56(0.05) \mathrm{abcd}$ & $1.09(0.04) \mathrm{cd}$ & $0.54(0.02)$ bcdef & $3.59(0.38) \mathrm{bcd}$ & $0.0197(0.0017)$ bcde & $0.42(0.05) \mathrm{cd}$ & $0.73(0.05)$ abcd & $0.56(0.06)$ cde \\
\hline $\mathrm{W}$ & $1.62(0.03) \mathrm{ab}$ & $1.18(0.02) \mathrm{abcd}$ & $0.53(0.01)$ cdef & $4.01(0.14)$ abcd & $0.0230(0.001)$ abcd & $0.43(0.03) \mathrm{cd}$ & $0.72(0.03) \mathrm{abcd}$ & $0.56(0.03)$ cde \\
\hline$\hat{Y}$ & $1.49(0.03)$ abcde & $1.18(0.02) \mathrm{abcd}$ & $0.56(0.01)$ abcdef & $3.86(0.14)$ abcd & $0.0198(0.001)$ bcde & $0.52(0.03) \mathrm{bcd}$ & $0.75(0.03) \mathrm{abc}$ & $0.70(0.03)$ abcde \\
\hline
\end{tabular}

Notes: A, Mengla; B, Yuanyang; C, Mojiang; D, Jinghong; E, Xichou; F, Zhenyuan; G, Tengchong; H, Jinggu; I, Ruili; J, Fengqing; K, Pingbian; L, Jiangcheng; M, Shuangjiang; N, Lancang; O, Lingyun; P, Longzhou; Q, Donglan; R, Tianlin; S, Debao; T, Tiane; U, Pingguo; V, Baise; W, Tianyang; X, Jingxi; Y, Napo; MADBH, mean annual increment of diameter at breast height; $\mathrm{MAH}$, mean annual increment of tree height; HCB.Height ${ }^{-1}$, rate of height to live crown base to tree height; CW, crown width; MAVOL, mean annual increment of stem volume; SF, stem form; CS, crown shape; BRA, branchiness. Means with standard error in parenthesis were of significant difference at 0.05 level according to Duncan's multiple range tests if followed by wholly different small letters in the same row. 
Table A5. Growth and quality traits for 25 provenances of Betula alnoids at Changning site.

\begin{tabular}{|c|c|c|c|c|c|c|c|c|}
\hline Provenances & MADBH $\left(\mathrm{cm} \cdot\right.$ year $^{-1}$ ) & MAH (m·year-1) & HCW.Height ${ }^{-1}$ & CW (m) & MAVOL $\left(\mathrm{m}^{3} \cdot\right.$ year $\left.^{-1}\right)$ & SF & CS & BRA \\
\hline A & $0.76(0.04) \mathrm{abcd}$ & $0.74(0.06)$ cde & $0.56(0.05) \mathrm{bcd}$ & $3.39(0.37) b c$ & $0.0017(0.0002)$ abcd & $0.85(0.05) \mathrm{a}$ & $0.78(0.07)$ & $0.78(0.08)$ \\
\hline B & $0.86(0.05) \mathrm{a}$ & $0.91(0.07) \mathrm{a}$ & $0.47(0.03) \mathrm{d}$ & $4.08(0.42) \mathrm{abc}$ & $0.0026(0.0005) \mathrm{ab}$ & $0.80(0.07) \mathrm{ab}$ & $0.63(0.14)$ & $0.77(0.08)$ \\
\hline $\mathrm{C}$ & $0.82(0.04) \mathrm{ab}$ & $0.87(0.04) \mathrm{abcd}$ & $0.51(0.02) \mathrm{bcd}$ & $4.79(0.28) \mathrm{ab}$ & $0.0024(0.0003) \mathrm{abc}$ & $0.76(0.06) \mathrm{ab}$ & $0.65(0.07)$ & $0.69(0.06)$ \\
\hline $\mathrm{D}$ & $0.74(0.03) \mathrm{abcd}$ & $0.82(0.04)$ abcde & $0.52(0.02) \mathrm{bcd}$ & $3.13(0.29) \mathrm{c}$ & $0.0018(0.0002)$ abcd & $0.70(0.06) \mathrm{ab}$ & $0.67(0.06)$ & $0.71(0.06)$ \\
\hline $\mathrm{E}$ & $0.81(0.03) \mathrm{abc}$ & $0.90(0.04) \mathrm{ab}$ & $0.50(0.02) \mathrm{cd}$ & $3.54(0.33) \mathrm{abc}$ & $0.0023(0.0002) \mathrm{abc}$ & $0.81(0.05) \mathrm{ab}$ & $0.67(0.05)$ & $0.78(0.05)$ \\
\hline $\mathrm{F}$ & $0.80(0.05) \mathrm{abc}$ & $0.81(0.03)$ abcde & $0.47(0.02) \mathrm{d}$ & $3.79(0.46) \mathrm{abc}$ & $0.0020(0.0003)$ abcd & $0.87(0.05) \mathrm{a}$ & $0.81(0.07)$ & $0.70(0.08)$ \\
\hline G & $0.77(0)$ abcd & $0.83(0)$ abcde & $0.51(0) \mathrm{bcd}$ & $2.99(0.72) \mathrm{c}$ & $0.0020(0)$ abcd & $0.77(0) \mathrm{ab}$ & $0.65(0)$ & $0.73(0)$ \\
\hline $\mathrm{H}$ & $0.79(0.04) \mathrm{abc}$ & $0.85(0.05)$ abcde & $0.50(0.03) \mathrm{cd}$ & $3.60(0.40) \mathrm{abc}$ & $0.0020(0.0003) \mathrm{abcd}$ & $0.83(0.07)$ a & $0.68(0.09)$ & $0.83(0.07)$ \\
\hline I & $0.60(0.05) \mathrm{e}$ & $0.73(0.05) \mathrm{de}$ & $0.54(0.03) \mathrm{bcd}$ & $3.95(0.49) \mathrm{abc}$ & $0.0011(0.0002) \mathrm{d}$ & $0.79(0.06) \mathrm{ab}$ & $0.66(0.09)$ & $0.66(0.09)$ \\
\hline $\mathrm{J}$ & $0.75(0.04)$ abcd & $0.84(0.04)$ abcde & $0.56(0.03) \mathrm{bcd}$ & $4.08(0.35) \mathrm{abc}$ & $0.0019(0.0002)$ abcd & $0.81(0.05) \mathrm{ab}$ & $0.71(0.08)$ & $0.81(0.06)$ \\
\hline $\mathrm{K}$ & $0.77(0.05) a b c$ & $0.83(0.07)$ abcde & $0.59(0.05) \mathrm{b}$ & $4.99(0.72) \mathrm{a}$ & $0.0019(0.0003) \mathrm{abcd}$ & $0.84(0.05) \mathrm{a}$ & $0.59(0.12)$ & $0.75(0.09)$ \\
\hline $\mathrm{L}$ & $0.79(0.02) a b c$ & $0.83(0.03)$ abcde & $0.52(0.02) \mathrm{bcd}$ & $4.08(0.27) \mathrm{abc}$ & $0.0020(0.0002)$ abcd & $0.80(0.04) \mathrm{ab}$ & $0.64(0.05)$ & $0.73(0.05)$ \\
\hline M & $0.71(0.04)$ bcde & $0.78(0.04)$ abcde & $0.51(0.02) \mathrm{bcd}$ & $3.87(0.37) \mathrm{abc}$ & $0.0016(0.0002) \mathrm{bcd}$ & $0.67(0.06) \mathrm{ab}$ & $0.72(0.07)$ & $0.66(0.07)$ \\
\hline $\mathrm{N}$ & $0.77(0.03) a b c$ & $0.83(0.03)$ abcde & $0.50(0.02) \mathrm{cd}$ & $3.16(0.27) \mathrm{c}$ & $0.0020(0.0002)$ abcd & $0.73(0.05) \mathrm{ab}$ & $0.69(0.05)$ & $0.71(0.06)$ \\
\hline $\mathrm{O}$ & $0.71(0.03)$ bcde & $0.74(0.03)$ cde & $0.58(0.03) b c$ & $4.11(0.34) \mathrm{abc}$ & $0.0015(0.0002) \mathrm{cd}$ & $0.70(0.06) \mathrm{ab}$ & $0.61(0.08)$ & $0.71(0.07)$ \\
\hline $\mathrm{P}$ & $0.87(0.04) \mathrm{a}$ & $0.90(0.03) \mathrm{abc}$ & $0.48(0.02) \mathrm{d}$ & $4.85(0.36) \mathrm{ab}$ & $0.0027(0.0003) \mathrm{a}$ & $0.72(0.05) \mathrm{ab}$ & $0.64(0.06)$ & $0.67(0.06)$ \\
\hline $\mathrm{Q}$ & $0.72(0.04)$ abcd & $0.83(0.07)$ abcde & $0.52(0.05) \mathrm{bcd}$ & $3.50(0.48)$ abc & $0.0016(0.0003) \mathrm{abcd}$ & $0.70(0.13) \mathrm{ab}$ & $0.90(0.06)$ & $0.81(0.13)$ \\
\hline $\mathrm{R}$ & $0.82(0.03) a b c$ & $0.87(0.03) \mathrm{abcd}$ & $0.51(0.02) \mathrm{bcd}$ & $3.47(0.24) b c$ & $0.0023(0.0002) \mathrm{abc}$ & $0.77(0.04) \mathrm{ab}$ & $0.69(0.05)$ & $0.76(0.04)$ \\
\hline $\mathrm{s}$ & $0.63(0.06) \mathrm{de}$ & $0.69(0.06) \mathrm{e}$ & $0.69(0.10) \mathrm{a}$ & $3.12(0.42) \mathrm{c}$ & $0.0011(0.0003) \mathrm{d}$ & $0.59(0.08) \mathrm{b}$ & $0.55(0.11)$ & $0.66(0.11)$ \\
\hline $\mathrm{T}$ & $0.78(0.04) \mathrm{abc}$ & $0.84(0.05)$ abcde & $0.49(0.03) \mathrm{cd}$ & $4.83(0.44) \mathrm{ab}$ & $0.0020(0.0003) \mathrm{abcd}$ & $0.70(0.10) \mathrm{ab}$ & $0.55(0.12)$ & $0.55(0.11)$ \\
\hline U & $0.71(0.03)$ bcde & $0.81(0.04)$ abcde & $0.52(0.02) \mathrm{bcd}$ & $4.27(0.32) \mathrm{abc}$ & $0.0017(0.0002) \mathrm{abcd}$ & $0.78(0.05) \mathrm{ab}$ & $0.58(0.06)$ & $0.77(0.06)$ \\
\hline $\mathrm{V}$ & $0.77(0.05) \mathrm{abcd}$ & $0.84(0.04)$ abcde & $0.47(0.02) \mathrm{d}$ & $3.95(0.44) \mathrm{abc}$ & $0.0020(0.0003)$ abcd & $0.82(0.06) \mathrm{ab}$ & $0.69(0.09)$ & $0.81(0.07)$ \\
\hline W & $0.84(0.03) \mathrm{ab}$ & $0.86(0.03) \mathrm{abcd}$ & $0.49(0.02) \mathrm{cd}$ & $3.93(0.32) \mathrm{abc}$ & $0.0023(0.0002) \mathrm{abc}$ & $0.76(0.06) \mathrm{ab}$ & $0.56(0.07)$ & $0.68(0.07)$ \\
\hline$x$ & $0.68(0.03)$ cde & $0.75(0.03)$ bcde & $0.48(0.01) \mathrm{d}$ & $4.04(0.30) \mathrm{abc}$ & $0.0014(0.0002) \mathrm{cd}$ & $0.71(0.05) \mathrm{ab}$ & $0.49(0.06)$ & $0.74(0.05)$ \\
\hline Y & $0.84(0.03) \mathrm{ab}$ & $0.87(0.03) \mathrm{abcd}$ & $0.50(0.02) \mathrm{cd}$ & $3.47(0.29) b c$ & $0.0024(0.0003) \mathrm{abc}$ & $0.85(0.03) \mathrm{a}$ & $0.69(0.06)$ & $0.76(0.05)$ \\
\hline
\end{tabular}

Notes: A, Mengla; B, Yuanyang; C, Mojiang; D, Jinghong; E, Xichou; F, Zhenyuan; G, Tengchong; H, Jinggu; I, Ruili; J, Fengqing; K, Pingbian; L, Jiangcheng; M, Shuangjiang; N, Lancang; O, Lingyun; P, Longzhou; Q, Donglan; R, Tianlin; S, Debao; T, Tiane; U, Pingguo; V, Baise; W, Tianyang; X, Jingxi; Y, Napo; MADBH, mean annual increment of diameter at breast height; $\mathrm{MAH}$, mean annual increment of tree height; $\mathrm{HCB} \cdot \mathrm{Height}^{-1}$, rate of height to live crown base to tree height; $\mathrm{CW}$, crown width; MAVOL, mean annual increment of stem volume; $\mathrm{SF}$, stem form; CS, crown shape; BRA, branchiness. Means with standard error in parenthesis were of significant difference at 0.05 level according to Duncan's multiple range tests if followed by wholly different small letters in the same row. 


\section{References}

1. Zeng, J.; Wang, Z.; Zhou, S.; Bai, J.; Zheng, H. Allozyme variation and population genetic structure of Betula alnoides from Guangxi, China. Biochem. Genet. 2003, 41, 61-75. [CrossRef] [PubMed]

2. Wang, C.S.; Zhao, Z.G.; Hein, S.; Zeng, J.; Schuler, J.; Guo, J.J. Effect of planting density on knot attributes and branch occlusion of Betula alnoides under natural pruning in southern China. Forests 2015, 6, 1343-1361. [CrossRef]

3. Sur, T.K.; Pandit, S.; Battacharyya, D.; Kumar, C.K.A.; Lakshmi, S.M.; Chatttopadhyay, D.; Mandal, S.C. Studies on the anti-inflammatory activity of Betula alnoides bark. Phytother. Res. 2002, 16, 669-671. [CrossRef] [PubMed]

4. Raj, A.D.A.; Malarvili, T.; Velavan, S. Restorative effect of Betula alnoides bark on hepatic metabolism in high fat diet fed Wistar rats. Int. J. Pharma Biol. Sci. 2015, 6, 1281-1288.

5. Zeng, J.; Guo, W.F.; Zhao, Z.G.; Weng, Q.J.; Yin, G.T.; Zhen, H.S. Domestication of Betula alnoides in China: Current Status and Perspectives. For. Res. 2006, 19, 379-384. (In Chinese with English Abstract)

6. Zeng, J.; Zheng, H.; Weng, Q. Geographic distributions and ecological conditions of Betula alnoides in China. For. Res. 1999, 12, 479-484. (In Chinese with English Abstract)

7. Wang, C.S.; Hein, S.; Zhao, Z.G.; Guo, J.J.; Zeng, J. Branch occlusion and discoloration of Betula alnoides, under artificial and natural pruning. For. Ecol. Manag. 2016, 375, 200-210. [CrossRef]

8. Chen, G.B. Preliminary experimental report on Betula alnoides provenance and family in Zhangzhou, Fujian. J. Fujian For. Sci. Technol. 2005, 32, 78-81. (In Chinese with English Abstract)

9. Guo, W.F.; Zeng, J.; Li, M. Provenance and family trials for Betula alnoides in Pingxiang Guangxi province I. early variation of growth traits. For. Res. 2008, 21, 652-656. (In Chinese with English Abstract)

10. Lin, W.F. The growth diffence for different family of Betula alnoides in Wutaishan. Guangdong For. Sci. Technol. 2008, 24, 16-21. (In Chinese with English Abstract)

11. Yang, Y.P.; Guo, J.J.; Huang, J.C.; Zhao, Z.G.; Zhou, Z.M.; Zeng, J. Early choose of Betula alnoides provenance and family in western part of Yunnan province. Seed 2012, 31, 67-70. (In Chinese with English Abstract)

12. Li, Y.J.; Suontama, M.; Burdon, R.D.; Dungey, H.S. Genotype by Environment Interactions in Forest Tree Breeding: Review of Methodology and Perspectives on Research and Application. Tree Genet. Genomes 2017, 13, 60. [CrossRef]

13. Costa, S.J.; Potts, B.; Dutkowski, G. Genotype by environment interaction for growth of Eucalyptus globulus in Australia. Tree Genet. Genomes 2006, 2, 61-75. [CrossRef]

14. Li, B.; McKeand, S.E. Stability of loblolly pine families in the southeastern US. Silvae Genet. 1989, 38, 96-101.

15. Kim, I.S.; Kwon, H.Y.; Ryu, K.O.; Choi, W.Y. Provenance by site interaction of Pinus densiflora in Korea. Silvae Genet. 2008, 57, 131-139. [CrossRef]

16. Zhao, X.Y.; Bian, X.Y.; Li, Z.X.; Wang, X.W.; Yang, C.J.; Liu, G.F.; Jiang, J.; Kentbayev, Y.; Kentbayeva, B.; Yang, C.P. Genetic stability analysis of introduced Betula pendula, Betula kirghisorum, and Betula pubescens families in saline-alkali soil of northeastern China. Scand. J. For. Res. 2014, 29, 639-649. [CrossRef]

17. Falkenhagen, E. A comparison of the AMMI method with some classical statistical methods in provenance research: The case of the south African Pinus radiata trail. For. Genet. 1996, 3, 81-87.

18. Osorio, L.F.; White, T.L.; Huber, D.A. Age trends of heritabilities and genotype-by-environment interactions for growth traits and wood density from clonal trials of Eucalyptus grandis Hill ex Maiden. Silvae Genet. 2001, $50,30-37$.

19. Suontama, M.; Low, C.B.; Stovold, G.T.; Miller, M.A.; Fleet, K.R.; Li, Y.; Dungey, H.S. Genetic parameters and genetic gains across three breeding cycles for growth and form traits of Eucalyptus regnans in New Zealand. Tree Genet. Genomes 2015, 11, 1-14. [CrossRef]

20. Wang, C.S.; Zhan, Z.G.; Zeng, J.; Guo, J.J.; Sha, E.; Guo, W.F.; Zeng, J.; Zheng, H.S. Relationship between planting density and tree growth process of Betula alnoides mid-young plantations in Pingxiang, Guangxi. For. Res. 2013, 26, 257-262. (In Chinese with English Abstract)

21. Wang, H.; Guo, J.J.; Zhang, D.; Li, Y.F.; Wang, C.S.; Zhao, Z.G.; Zeng, J. Growth, form variation and early selection of hybrid clones of birch in Betula section betulaster. Mol. Plant Breed. 2018, 16, 1638-1646. (In Chinese with English Abstract) 
22. Merk, H.L.; Yarnes, S.C.; Van Deynze, A.; Tong, N.K.; Menda, N.; Mueller, L.A.; Mutschler, M.A.; Loewen, S.A.; Myers, J.R.; Francis, D.M. Trait diversity and potential for selection indices based on variation among regionally adapted processing tomato germplasm. J. Am. Soc. Hortic. Sci. 2012, 137, 427-437. [CrossRef]

23. Lai, M.; Dong, L.M.; Yi, M.; Sun, S.W.; Zhang, Y.Y.; Fu, L.; Xu, Z.H.; Lei, L.; Leng, C.H.; Zhang, L. Genetic variation, heritability and genotype $\times$ environment interactions of resin yield, growth traits and morphologic traits for Pinus Elliottii at three progeny trials. Forests 2017, 8, 409. [CrossRef]

24. Liu, Q.H.; Zhou, Z.C.; Fan, H.H.; Liu, Y.R. Genetic variation and correlation among resin yield, growth, and morphologic traits of Pinus massoniana. Silv. Genet. 2013, 62, 38-44. [CrossRef]

25. Diao, S.; Hou, Y.; Xie, Y.; Sun, X. Age trends of genetic parameters, early selection and family by site interactions for growth traits in Larix Kaempferi open-pollinated families. BMC Genet. 2016, 17, 104. [CrossRef]

26. Yan, W.; Hunt, L.A.; Sheng, Q.; Szlavnics, Z. Cultivar evaluation and mega-environment inverstigation based on the GGE Biplot. Crop. Sci. 2000, 40, 597-605. [CrossRef]

27. Yan, W. GGEbiplot-A windows application for graphical analysis of multienvironment trial data and other types of two-way data. Agron. J. 2001, 93, 1111-1118. [CrossRef]

28. Baltunis, B.S.; Brawner, J.T. Clonal stability in Pinus Radiata across New Zealand and Australia. I. Growth and form traits. New For. 2010, 40, 305-322. [CrossRef]

29. Pliura, A.; Zhang, S.Y.; MacKay, J.; Bousquet, J. Genotypic variation in wood density and growth traits of poplar hybrids at four clonal trials. For. Ecol. Manag. 2007, 238, 92-106. [CrossRef]

30. Ivković, M.; Gapare, W.J.; Yang, H.X.; Dutkowski, G.; Buxton, P.; Wu, H. Pattern of genotype by environment interaction for radiata pine in southern Australia. Ann. For. Sci. 2015, 72, 391-401. [CrossRef]

31. Dungey, H.S.; Low, C.B.; Lee, J.; Miller, M.A.; Fleet, K.; Yanchuk, A.D. Developing breeding and deployment options for Douglas-fir in New Zealand: Breeding for future forest conditions. Silvae Genet. 2012, 61-63, 104-115. [CrossRef]

32. Gapare, W.J.; Ivković, M.; Dutkowski, G.W.; Spencer, D.J.; Buxton, P.; Wu, H.X. Genetic parameters and provenance variation of Pinus radiata D. Don. 'Eldridge collection' in Australia 1: Growth and form traits. Tree Genet. Genomes 2012, 8, 391-407. [CrossRef]

33. Ramesh, K.J. A Study of Variability, Associations, and path analysis in poplar (Populus deltoides Bartr. ex Marsh). J. Sustain. For. 2012, 31, 185-204.

34. Stener, L.G.; Hedenberg, O. Genetic Parameters of wood, fibre, stem quality and growth traits in a clone test with Betula Pendula. Scand. J. For. Res. 2010, 18, 103-110. [CrossRef]

35. Wang, H.; Zeng, J.X.; Luo, B.G.; Guo, J.J.; Wang, C.S.; Zhao, Z.G.; Zeng, J. Multiple-trait combined selection of superior Betula alnoides clones in eastern Guangdong. J. Cent. South Univ. For. Technol. 2017, 37, 72-75. (In Chinese with English Abstract)

36. Flores, F.; Moreno, M.T.; Cubero, J.I. A comparison of univariate and multivariate methods to analyze GXE interaction. Field Crop. Res. 1998, 56, 271-286. [CrossRef]

37. Liziniewicz, M.; Berlin, M. Differences in growth and areal production between Norway spruce (Picea abies L. Karst) regeneration material representing different levels of genetic improvement. For. Ecol. Manag. 2019, 435, 158-169. [CrossRef]

38. Grattapaglia, D.; Ribeiro, V.J.; Rezende, G.D.S.P. Retrospective selection of elite parent trees using paternity testing with microsatellite markers: An alternative short term breeding tactic for Eucalyptus. Theor. Appl. Genet. 2004, 109, 192-199. [CrossRef]

(C) 2019 by the authors. Licensee MDPI, Basel, Switzerland. This article is an open access article distributed under the terms and conditions of the Creative Commons Attribution (CC BY) license (http://creativecommons.org/licenses/by/4.0/). 